\title{
Botanical geography correspondence between Alexander von Humboldt and Filippo Parlatore (1851-1852)
}

\author{
Enrico Banfi ${ }^{*}$, Agnese Visconti ${ }^{2}$
}

\begin{abstract}
This paper focuses on the relationship between Alexander von Humboldt, the famous German explorer-naturalist and Filippo Parlatore, botanist from Palermo (Sicily, Italy) in the field of botanical geography. Our considerations are based on three letters written by Humboldt to Parlatore in May 1851 and two letters with attachments written by Parlatore in answer to Humboldt in May 1851 and June 1852. The former are preserved in the Biblioteca comunale di Palermo (Palermo City Library) and the latter in the Staatsbibliothek zu Berlin (Berlin State Library).

On reading the correspondence it can be inferred how, on the one hand, Humboldt asked Parlatore for verification on some important questions of botanical geography that he intended to cover in the second part (never published) of volume five of his Kosmos, and how, on the other hand, Parlatore diverged in his answers from Humboldt's intent to search for universal laws to explain the distribution of plants on the planet. In fact, Parlatore was engaging in new lines of research, which, though stemming from Humboldt, were moving towards a modern twodimensional interpretation of natural plant communities according to which vegetation and flora, though interacting, are distinct realities and require different methods of study.

The paper includes a portrait of Parlatore and one of Humboldt, a taxonomic table of the plants mentioned by the two scientists in their correspondence and illustrations of a selection of the same plants.
\end{abstract}

Key words: flora, Herbarium Centrale Italicum, XIX Century, phytogeography, vegetation.

Riassunto - Corrispondenza geobotanica tra Alexander von Humboldt e Filippo Parlatore (1851-1852).

L'articolo sviluppa riflessioni sui rapporti tra il famoso esploratorenaturalista tedesco Alexander von Humboldt e il botanico palermitano Filippo Parlatore nel campo della geografia botanica. Esso si basa su tre lettere (conservate nella Biblioteca comunale di Palermo) scritte da Humboldt a Parlatore nel maggio del 1851 e su due lettere (conservate nella Staatsbibliothek zu Berlin) corredate di altrettanti allegati, inviate da quest'ultimo nel maggio 1851 e nel giugno 1852 in risposta alle precedenti.

\footnotetext{
Collaboratore volontario Sezione di Botanica, Museo di Storia Naturale di Milano, Corso Venezia 55, 20121 Milano, Italia.

${ }^{2}$ Via Podgora 3, 20122 Milano, Italia.

E-mail: visconti.agnese@gmail.com

* Corresponding author: parajubaea@gmail.com

(C) 2020 Enrico Banfi, Agnese Visconti
}

Received: 28 July 2020

Accepted for publication: 20 October 2020

Online publication: 18 November 2020
Dalla lettura del carteggio si evince, da un lato come Humboldt richiedesse a Parlatore il riscontro ad alcune importanti questioni di geografia botanica che intendeva trattare nella seconda parte (rimasta inedita) del quinto volume del suo Kosmos, e dall'altro come Parlatore nelle sue risposte divergesse dall'intento di Humboldt di ricercare per la distribuzione delle piante sul pianeta leggi di carattere universale, e partecipasse invece alle nuove linee di ricerca che, pur prendendo le mosse da Humboldt, si stavano indirizzando verso l'odierna interpretazione a due dimensioni delle comunità naturali di piante, in base alla quale vegetazione e flora sono realtà interagenti ma distinte e richiedono metodi di studio differenti.

Corredano il lavoro un ritratto di Parlatore e uno di Humboldt, un prospetto tassonomico delle piante citate dagli autori e alcune illustrazioni di piante scelte tra quelle citate nel carteggio.

Parole chiave: XIX secolo, fitogeografia, flora, Herbarium Centrale Italicum, vegetazione.

\section{INTRODUCTION}

Before examining the correspondence between the famous German scientist-explorer Alexander von Humboldt (1769-1859) and the Sicilian botanist Filippo Parlatore (1816-1877; Fig.1), which is published here, it is necessary to clarify what is currently known regarding the relationship between the two naturalists in the field of phytogeography in order to demonstrate its importance for the history of botany. Alexander von Humboldt established phytogeography as a new scientific discipline, aimed at researching the relationships between plants and the climate ${ }^{1}$, during his travels in the tropical regions of America (1799-1804), travels which had the scope of studying the interconnections between natural phenomena on the Earth's surface (Humboldt, 1805 : V-VI).

In general, historians of botany have mentioned the relationship between the two scientists with the intention, essentially, of honouring the figure of the Sicilian scientist by placing him alongside the great German explorer (Haynald, 1879; Tirrito, 1882: 15; Negri, 1927; Rodolico, 1997: 58; Moggi, 1978; Visconti, 1987; Visconti, 2004; Moggi, 2009). However, until now, no in-depth analysis has been performed concerning the scientific interrelations which began to develop between the two scholars during the first stay of the Sicilian botanist in Paris (March 1841-May 1842) and which deepened significantly during his subsequent meetings with the German scientist in Berlin in 1844 (Parlatore, 1992: 123) and in 1851 (Parlatore, 1992: 189-201). 
Insufficient documentation has so far hindered an accurate reconstruction and consequent interpretation of the relationship between Parlatore and Humboldt. This barrier has generally limited studies to a mere description of the role played by the German scientist in intervening with Leopold II, Grand Duke of Tuscany, in the nomination of Parlatore as professor of Botany and director of the newly-instituted Herbarium Centrale Italicum (27 September 1842) in the Museum of Physics and Natural History of Florence. There continues to be a lack of documentation even today and any attempt at a reliable and robust reconstruction is therefore hindered. Nevertheless, the documents presented here should enable some interesting headway to be made towards a deeper understanding of the geobotanical conceptions of the two scholars. In this sense, the letters constitute a small nucleus, which

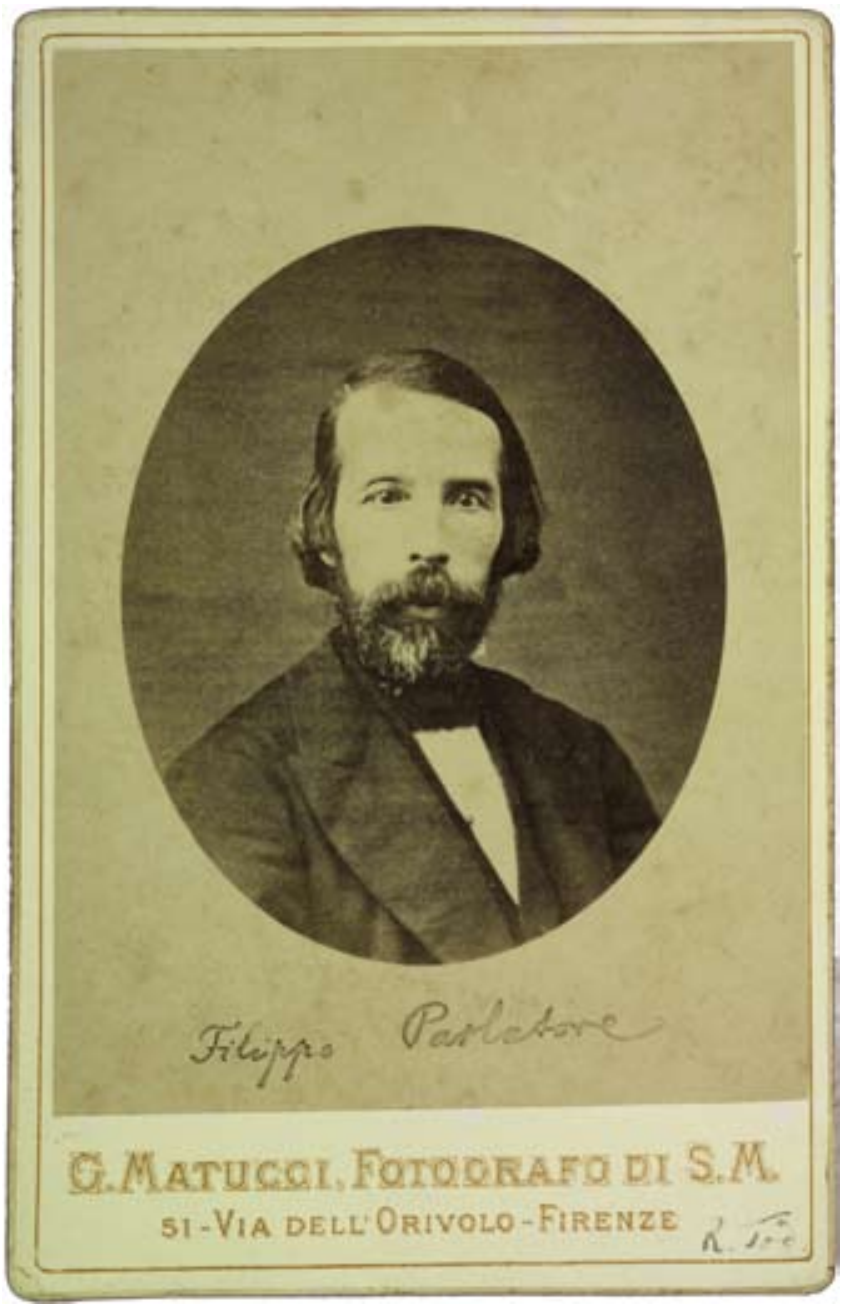

Fig. 1 - Filippo Parlatore (1816-1877) in a photographic portrait by Gustavo Matucci (187?). Handwritten title on the recto, where the following note also appears: R. Tod. [Todaro Collection]. Mounted on $165 \times 105 \mathrm{~mm}$ cardboard. 1 photo: albumin; 137 x $97 \mathrm{~mm}$. University of Padua. Library of the Botanical Garden (IB.FF.8). Creative Commons license CC BY-NC-SA 4.0. / Filippo Parlatore (1816-1877) in un ritratto fotografico di Gustavo Matucci (187?). Titolo manoscritto sul recto, dove compare anche la nota: $\mathrm{R}$. Tod. [Raccolta Todaro]. Montata su cartoncino $165 \times 105 \mathrm{~mm}$. 1 fotografia : albumina ; 137 x $97 \mathrm{~mm}$. Università di Padova. Biblioteca dell'Orto Botanico (IB.FF.8). Licenza Creative Commons CC BY-NC-SA 4.0. provides several indicators on the basis of which we can attempt to develop our considerations.

Firstly, regarding Parlatore's stay in Paris in 1841-1842. It is known that, having left Palermo in October 1840 (Parlatore, 1992: 67) - encouraged by Theodor von Heldreich, the curator of the herbarium of the Genevan botanist Augustin Pyrame de Candolle, who was collecting herbs in Sicily in the summer of that year (Heldreich, 1843; Parlatore, 1992: 108, Halácsy, 1902) - he reached the French capital in the spring of 1841 (Parlatore, 1992: 88). He had stayed before some weeks in Geneva (Parlatore, 1992: 84-86) where, for the first time, he was able to approach the study of botanical geography according to the innovative methods developed by Candolle. By extending Humboldt's initial effectual, descriptive view based on the relation between vegetation and climate, which envisaged the latter as a cause of the former (Béguinot, 1906; Tomaselli, 1956: 20-21), Candolle was attempting to formulate models which regulate plant distribution by including a great variety of factors, other than temperature, such as historic, edaphic, pedological, etc. (Candolle A. P. de, 1840 and 1844). It was on Candolle's method of researching and interconnecting the diverse elements which contribute to the formation of plant associations and their distribution that Parlatore was to found his phytogeographic perspective, as is apparent in his letters to Humboldt, in particular in relation to the enclosure to the letter of June 1852 (Letter 5).

Nevertheless, Candolle's instruction was not to last long and Parlatore decided to leave for Paris, probably because of the Swiss botanist's serious illness. In Paris, he attended lessons in the most eminent scientific institutions and began to establish relationships with the most renowned naturalists of the time, including Humboldt ${ }^{2}$ himself. The little documentation in our possession does not tell us whether the German scientist provided Parlatore with any indication of how he could further his geobotanical studies, nor it is known whether Humboldt instructed the Sicilian botanist during their meeting in Berlin in 1844 (Parlatore, 1992: 123). However, on the basis of the relationship between the two naturalists that emerges from the correspondence reproduced here, it can be assumed that Humboldt gave Parlatore some basic tutoring. In this respect, it is possible to begin from Parlatore's report on his alpine travels (Parlatore, 1850), a work focusing on the study of plants in relation to a given region. In the introduction, the Sicilian scientist states that "[his] intention was to study the colossal Alpine range from the point of view of botanical geography in order to become familiar with its physiognomic variations and so determine the range limits of plants at the various elevations of those mountains" (Parlatore, 1850: V). He then goes on to describe the ascent of Mont Crammont, obviously taking by way of example, since both are isolated mountains, Humboldt's excursion to the summit of Pic Teide, a volcano on the island of Tenerife. Still in the German scientist's footsteps, Parlatore proceeds in his ascent to the summit, subdividing the territory he is considering into distinct zones identified on the basis of the relation between the vegetational groups, the altitude and the temperature. Thus, while for the Pic Teide those zones were named by Humboldt as: of the vine, of the laurel, of the pine, of the Retama and of the grasses, "placed, as if in layers, one 
above the other" (Humboldt, 1814: 182), for Mont Crammont they were named by Parlatore as: of the chestnut, of the walnut, of the pine, of the pasture grasses at a higher elevation than the pine, and of the grasses at the summit of the mountain (Parlatore, 1850: 4-5, 9-10,14-19, 23-24). However, differently from Humboldt's text, the Sicilian botanist's writings introduce the need to collect many "facts which must then be correlated with the meteorological conditions of the various countries" (Parlatore, 1850: 50 and 53). It is clear from these words that, while Parlatore had followed Humboldt's example for the starting point of his studies, he meant to continue along the path suggested by Candolle, in whose regard Humboldt, on the contrary as shall be seen, was quite disdainful.

With his return from Mont Crammont, Parlatore had definitely become an independent, mature scientist, ready to apply the study of the diverse factors involved in the formation of plant associations and their distribution, and also to face a real expedition which would allow him to extend his studies of the vegetation of Mont Crammont to the vegetation of vaster regions. Hence the decision, taken in the spring of 1851 (Parlatore, 1992: 188-189), to travel to the Scandinavian peninsula ${ }^{3}$ with the aim of "applying [his] thoughts on studying the form and nature of plants in different regions of the earth for the truly important studies of botanical geography which, even more than in the past, [his] mind had been set on over the last two years" (Parlatore, 1992: 188); and, indicative of the scientific independence he had also reached, the resolve, once he arrived in Stockholm (August), to change the itinerary of his journey and invert what had been suggested during his meetings with Humboldt in Berlin (May) (Parlatore, 1992: 193). Of these meetings, or better of the requests made to Parlatore on the part of Humboldt for clarification and information for the drafting of the geobotanical part of the last volume of the Kosmos Entwurf einer physischen Weltbeschreibung ${ }^{4}$, a grandiose treatise on the structure of the universe, there remain, as a trace, the words of Parlatore which reflect both the geobotanical interest of the two scientists and the opinions of Humboldt, who "spoke at length on botanical geography, not valuing particularly the works of de Candolle and Forbes ${ }^{6}$ in whom le souffle de la nature ${ }^{7}$ was missing, according to him." Parlatore adds that Humboldt "approved of [his] thoughts in that branch of science ${ }^{8}$ and because of this, glad to see [him] undertake a journey to the frozen parts of the earth which he had set his heart on but had never been able to undertake, promised and actually gave [him] a warm letter of introduction to King Oscar of Sweden" (Parlatore, 1992: 190). This suggests that the Sicilian botanist had become a precious interlocutor for the German naturalist. To corroborate this impression are the words of esteem written about Parlatore to the physician and botanist Christian Gottfried Ehrenberg by Humboldt himself who, in telling his friend of the presence in Berlin of the Sicilian botanist, describes him as a brilliant and intelligent (geistreich) person (Humboldt, [1851]). The letters between Humboldt and Parlatore, with their questions and answers, which are the subject of our considerations here, further improve the impression.

The first letter was delivered to Parlatore, who was already in Berlin, on 17 May (Letter 1) and the Sicilian botanist, torn between the preparations for his journey to the Scandinavian peninsula ${ }^{10}$ and the desire to acquiesce in what was asked of him, replied "as best [he] could" (Parlatore, 1992: 190) by sending a list of information on the geographic distribution of some tropical and Italian Cyperaceae (sedges) and Poaceae (grasses) taken almost exclusively from the first volume of his Flora italiana ${ }^{11}$ together with his notes on the second volume of that work, in print at the time (Letter 2 and enclosure).

A second letter was then sent by Humboldt on 20 May (Letter 3) in which the German scientist posed certain questions that bring out his geobotanical conception directed at attempting a formulation of universal laws ${ }^{12}$, based not so much on biological sciences but on a causeeffect perspective widely practised by him from the beginning of his studies and closer to the non-biological sciences. The Sicilian botanist was only able to respond to these questions after having carried out accurate and indepth verification in the Herbarium Centrale Italicum and among the volumes of the library of the Museum of Florence (as may be inferred in Letter 5). This second letter from Humboldt therefore remained unanswered for quite some time. In fact, Parlatore was only able to take it into consideration after more than a year, on recovering from a long convalescence following a paralysis caused by the fatigue, the worry and the privations suffered in the snow and ice of Scandinavia (Parlatore, 2012: 356-357).

And because still seriously ill, he was unable to answer - except with a simple promise, sent in January 1852 (as may be inferred in Letter 5) - to the request sent again to him in November 1851 by Humboldt (Letter 4), who, with his good wishes and encouragement to return to his studies, also sent Parlatore a lithograph from a watercolour by Eduard Hildebrandt (1848; Fig. 2) portraying himself at his desk engaged in writing volume two of the $\operatorname{Kosmos}^{13}$.

A request and a gift which were only to reach their goal at the end of June 1852 when Parlatore, completely recovered, was able to reply in an exhaustive and precise manner (Letter 5 and enclosure) to Humboldt's letter of 20 May (Letter 3), demonstrating how he had complete mastery of the geobotanical issues.

As for Humboldt, it is clear that in his letters he was asking for clarification with a view to writing the second part of the last volume of the Kosmos, in which he intended to present a geobotanical synthesis grounded prevalently in fixed relationships between climate and plants in an attempt to reconduct the illimitable variety of plants to universal concepts. However, his proposition did not take into account the more complex views that in those years were moving the minds of scholars in biological disciplines towards perspectives increasingly engaged in identifying and including the innumerable internal and external factors that condition a living organism and give rise to the habitat of a particular species, without wanting to infer universal systems. The climate, just as Humboldt had envisioned, was to remain a supporting pillar or, better, the starting point of successive geobotanical research, but oriented at considering a plant not so much as an effect of the climate but rather "as an expression of the most diverse and complicated movements of inorganic nature to which it has to adapt" (Grisebach, 1872: V). 
There are some issues of particular interest in relation to Humboldt and Parlatore's different geobotanical conceptions, which can be commented on briefly. Firstly, the request for confirmation on the part of the German naturalist on the possibility of establishing a correlation between the plants of the most southern zones of South America and those of the most northern part of North America and Europe. Parlatore responds to this question by reflecting on how the floristic composition of a region cannot be regulated by laws or correspond to an absolute concept but can be interpreted by taking into account the species occurring there naturally and those introduced by human action (enclosure with Letter 5). Nor is it possible, according to Parlatore, to affirm unequivocally that there exist plants that are common to all the mountainous regions of the earth (enclosure with Letter 5). It is also in the belief that there are cosmopolitan plants on which Humboldt questions the Sicilian botanist who responds by demonstrating the inconsistency of the question and inviting the German scientist to form a mental image in which similarities among European, Asian and American floras emerge at the North Pole and descend towards the Equator issuing longer and longer "rays of vegetation"14 which, by distancing themselves the one from the other, describe places on the earth's surface with completely differing floras (enclosure with Letter 5).

Unfortunately, it is currently impossible to know what Humboldt's thoughts were in relation to Parlatore's answers or how the scientific relations between the two scholars continued, if at all. However, in the light of the notes written by Humboldt on the first letter sent to him by Parlatore (Letter 2), we believe that the German scientist intended to focus on at least two phenomena which seemed to diverge from his convictions on climate and vegetation: a tropical plant that also grew on Ischia island (Italy) and the interruption of vegetation on the volcano Etna (Sicily, Italy) just above the Casa degli Inglesi, that is to say at an elevation inferior to that at which the vegetation ceases on Mount Blanc, situated at a higher latitude.

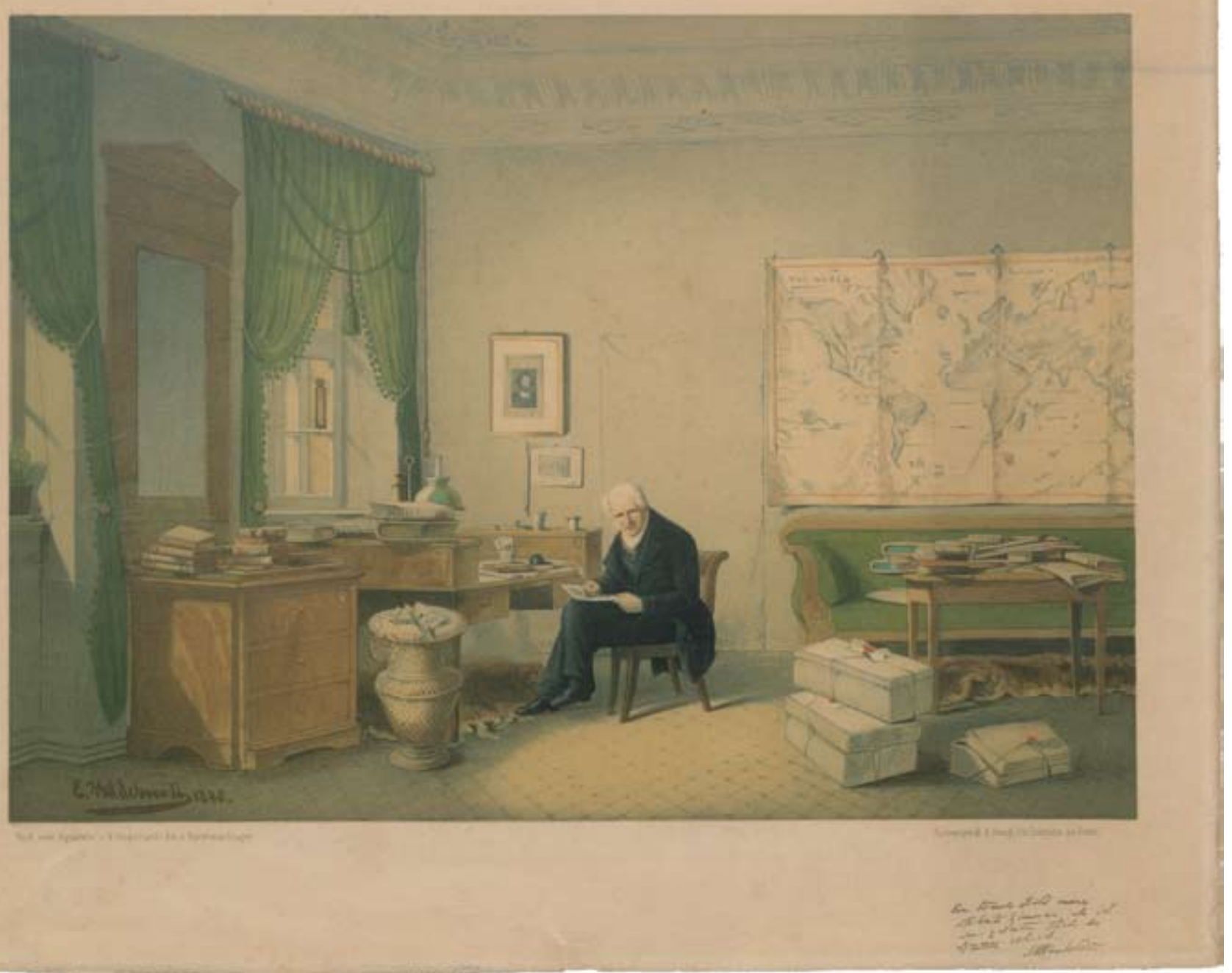

Fig. 2 - Alexander von Humboldt in his studio (1848). Lithograph by J. Bardtenschlager from a watercolor by Eduard Hildebrandt. / Alexander von Humboldt nel suo studio (1848). Litografia di J. Bardtenschlager da un acquerello di Eduard Hildebrandt. $32,30 \mathrm{~cm}$ x 41,80 cm. Inv.-Nr.: HU 01/8 DR. C Courtesy of Stiftung Stadtmuseum Berlin. 


\section{NOTE ON THE CORRESPONDENCE}

It is not known precisely when the correspondence between Humboldt and Parlatore began but it is thought to have been only occasional, if there had been any at all, before the period considered here since no other letters have been found prior to those presented here.

During Parlatore's stay in Paris, it is probable that Humboldt invited the botanist from Palermo to participate in some scientific discussions organised at his home and that Parlatore received verbal indications directly from Humboldt as to his future as a botanist, without there necessarily being any correspondence between the two. Successively, after Parlatore was called to Florence, the two scholars may have exchanged written courtesies, in particular when the Sicilian botanist sent his works to Humboldt (Stevens, [1863]: 543-544); and also later perhaps, in view of their meetings in Berlin in 1844 (Parlatore, 1992: 123) and in 1851 (Parlatore, 1992: 190). Instead, it is known for certain that the brief but intense correspondence between the two scientists which is presented here began during this last visit.

The three letters in Humboldt's own handwriting (Letters 1, 3, 4), published by Francesco Rodolico in 1968 without any scientific annotation ${ }^{15}$, have been compared with the originals preserved in the Biblioteca comunale di Palermo (Palermo City Library) and transcribed here in full with the addition of the necessary historical-botanical observations and notes.

Parlatore's letters (Letters 2 and 5), which are held in the Staatsbibliothek zu Berlin (Berlin State Library), were transcribed from the digital versions available on the web site of the library itself. Both letters come with other documents in attachment. The first (Letter 2), still with the original envelope without an address, is undated and lacks the usual opening salutation: a sign that the letter could have been delivered through the city's postal service (petite poste, see Letter 1), as were the first two letters sent by Humboldt, when Parlatore was in Berlin. Notes in Humboldt's handwriting can be found in German, French, Italian and Latin on the envelope, the letter and a sheet of paper glued to the letter. As to the four sheets of paper attached, three are in Parlatore's handwriting with the following words at the bottom of the third sheet in Humboldt's handwriting: Parlatore Mai [May] 1851; the fourth is a blank sheet on which is written in Humboldt's hand: Géographie des plantes. From the contents of the enclosure it is clear that this letter is an answer to the one sent to Parlatore by the German naturalist on 17 May (Letter 1).

The second letter sent by Parlatore (Letter 5) is accompanied by an enclosure of six sheets written on both sides: this is the answer to the questions formulated by Humboldt in his letter of 20 May (Letter 3).

In the transcription of the letters, enclosures and notes, every possible effort has been made to reconcile the criterion of textual accuracy with the need to provide the reader with instruments useful for an understanding of the text. As a general rule, the principle of preservation was therefore adopted, except for actions on the most manifest spelling and grammatical mistakes, occasional punctuation, obvious oversights and occasional repeti- tions which were corrected or eliminated. Underlining and variations in spelling were preserved, as signs of the author's preference. Abbreviations have been reproduced in their original form, completed where this was thought necessary and the additions have been placed in square brackets.

No action was taken on the accepted forms of spelling for the times, even if different from current forms, as in the case of capital and small letters for the genus and the species names.

For greater clarity, the titles of the works quoted in all the letters and enclosures have been written in italics and any eventual abbreviated or missing words have been written in full where necessary, with the exception of the list of plants enclosed with Letter 2, in which Parlatore repeatedly and exclusively quotes volume I of his Flora italiana, abbreviating the title to: fl. ital. I or fl. it. I., of which, only the first mention is written in full as Flora italiana, vol. I.

The words which compose Humboldt's notes on Parlatore's Letter 2, on the sheet glued to the same letter and on the envelope have been reproduced in full where these were complete words, furnished with square brackets for the missing part of abbreviated words or for those of uncertain legibility, and replaced with three dots in square brackets if not legible.

The location and the shelfmark of the documents are indicated at the foot of each document. The website address of the digital copies has been included for the documents preserved in the Staatsbibliothek zu Berlin.

Moreover, we would like to point out that in Letter 5 of the correspondence in question, Parlatore mentions a letter sent to Humboldt in January 1852, but for which there is still no trace.

\section{NOTE ON THE TAXONOMIC TABLE OF THE PLANTS MENTIONED}

The table positioned at p. 34 reproduces the list of the taxa (families, tribes, genus names, species and infraspecific names) quoted by Humboldt and Parlatore in their correspondence and annotated by Humboldt in Letter 2. The table is structured in four columns: Column 1 contains the original name given by the author; Column 2 , the family to which the plant belongs; Column 3 , its currently accepted name; Column 4 , the reference to the original name. In relation to the nomenclature, it must be specified that the names in Column 1 were not subject to the regulations now in force which are based on an international code (ICN: Turland et al., 2018) instituting how to use the names, how to choose them in accordance with the principle of rank and other criteria, how to write them (orthography), how to validate the publication of a new taxon, how to adhere to the nomina conservanda and numerous other rules. At that time, the botanical world, as far as names were concerned, advanced according to a kind of collective common sense, by which the names circulated in their most fashionable versions, often irrespective of rank or original spelling, ambiguous in the use of terminations (-dendron/-dendrum, -coccon/-coccum, -gyna/-gyne etc.) and inattentive to 
grammatical agreement (Heteropogon contortum instead of contortus, Onosma arenarium for arenaria etc.). Reference to the authors of the name (describer/s or eventual revisor/s) was often imprecise or erroneous because of confusion between the describer and the revisor or vice-versa. Sometimes, the name of a species was even attributed to describers of floras, to taxonomic editors and to compilers of horticultural catalogues who were only reproducing the names of species already described by others. Column 1 also includes non-scientific names and expressions, for which an effort has been made to extract the taxonomic information reproduced in Column 3. The official reference for the names of families is Stevens (2020). The nomenclature of the tribes follows Reveal J. L. (2012). The binomial nomenclature (genus names, species, infraspecific names) adheres to the IPNI (2020), Pignatti et al. (2017-2019), Bartolucci et al. (2018), Portal of the Flora of Italy (2020), as well as successive updates for single taxa.

Finally, the abbreviations in Column 4 refer to the original sources of information and more precisely to:

HP2: Humboldt's notes on Letter 2 from Parlatore and on the sheet glued to Letter 2;

HP2B: Humboldt's notes on the envelope of Letter 2 from Parlatore;

HP3: Humboldt, Letter 3;

P2A: Parlatore, enclosure with Letter 2;

P5A: Parlatore, enclosure with Letter 5.

\section{Letter 1}

[from Humboldt to Parlatore]

Combien j'ai regretté d'avoir manqué ce matin une si instructive conversation que la vôtre, Monsieur! Si vous n'allez pas demain a Potsdam voir jouer les eaux ${ }^{16}$, je vous demande en grâce de venir m'honorer d'une petite visite demain dimanche, à onze heures. Vous pourrez aussi choisir tout autre jour en me l'annonçant d'avance par la petite Poste.

Je voudrais profiter de vos lumières sur les Dicotylédones que Gullemin et Perrottet ont trouvées en Sénegambie ${ }^{17}$ et Robert Brown a vues dans les hérbiers du Congo $^{18}$, plantes Dicotylédones qui habitent les côtes du Brésil.

Je vous démanderais en grâce de me donner pour le Cosmos quelques lignes avant le mois de Septembre sur des problèmes analogues.

Je tiens aux identités certaines des latitudes tempérées identiques des zones tempérées au Nord et au Sud de l'Equateur, en séparant les graminées et les Cypéracées des Dycot[ylédones] des régions d'Afrique ou de l'Inde avec l'Amérique tropicale ${ }^{19}$.

Mille affectueux hommage! Je ne doute pas que le Roi $^{20}$ se fera un plaisir de vous accorder l'Aigle Rouge 3 eme classe ${ }^{21}$. Il faut le laisser revenir au repos

Ce samedi 17 Mai 1851

A. Humboldt

Biblioteca Comunale di Palermo, Fondo Parlatore, 5 Qq D $16(22 / 1)$
Letter 2 and Parlatore's handwritten enclosure, Humboldt's handwritten notes on the letter, his notes on the sheet glued to the letter, his notes on the envelope containing the letter

[Letter handwritten by Parlatore sent to Humboldt through a third party, probably Humboldt's valet, Johann Seifert. It bears the following penciled note perhaps also written by Seifert: Parlatore]

La Pteris longifolia, qui croit à Ischia, est une plante que de la région mediterranéenne s'etend jusque aux pays tropicaux oû elle est très répendue. Elle se trouve en Italie à Ischia près de Naples, à Malaga en Espagne, en Barberie, aux Iles Canariés, en Arabie, à l'Ile de France [Mauritius], aux Indes Orientales, en Chine, dans plusieurs iles tropicales de l'Océanie, dans l'Amerique tropicale en Columbie, aux iles de Cube, de Jamaique etc.

Je ne sais pas si Mr. le Baron de Humboldt trouvera de l'interet pour lui dans l'existence de Lycopodium cernuum dans une des iles Açoriques, plante du reste très rependue dans les pays tropicaux du vieux et du nouveau monde. Je pourrais plus tard envoyer à Mr. de Humboldt tous les details qui concernent les localités de cette plante dans les iles Acoriques, car je les ai reunis dans mes manuscrits de géographie botanique $^{22}$.

[Parlatore's handwritten enclosure]

Cyperacées qui se trouvent en Italie et dans les pays tropicaux

Cyperus globosus Allioni Dans les lieux humides près de Var à Nice. Fl[eurit] Juill[et] - Octobre vivace.

-Cette plante se trove aussi dans le midi de l'Éspagne près de Malaga, Toloz [Toulouse] et Cartama, en Orient, en Arabie, dans les Indes Orientales, dans l'Ile de France [Mauritius]

Cyperus polystachyos Rottboll -Dans l'ile d'Ischia près des fumaroli di Frasso et des Caciotti à un temperature atmospherique de 30 degrés, cette plante enfonce les racines dans le sol qui est à une temperature de 50 degrés. Ces temperatures ne sont pas observées par moi même mais je les rapporte après Mr. Tenore, qui n'indique pas même si elles son après le thermometre centigrade ou de Réaumur, il est probable qu'il se soit servi du therm. de Reaumur. Floraison de Juin à Octobre, vivace.

-C'est une éspece très repandue dans les pays tropicaux, d'oû elle s'avance dans les climats tempérés de deux hemisphères. Elle se trove en Europe seulment à l'ile d'Ischia, en Afrique près la Calle [El Kala] en Algérie, à Chama dans les royaumes d'Owar[e] [part of Ghana] et de Benin et au Cap de bonne ésperance, dans l'ile de France, en Arabie, dans les Iles Philippines, dans les Marianes et dans les iles de Sandwich, à Pondichey, en Chine, à la Nouvelle Hollande [Australia], dans l'Amerique du Sud au Brésil et à Montevideo. 


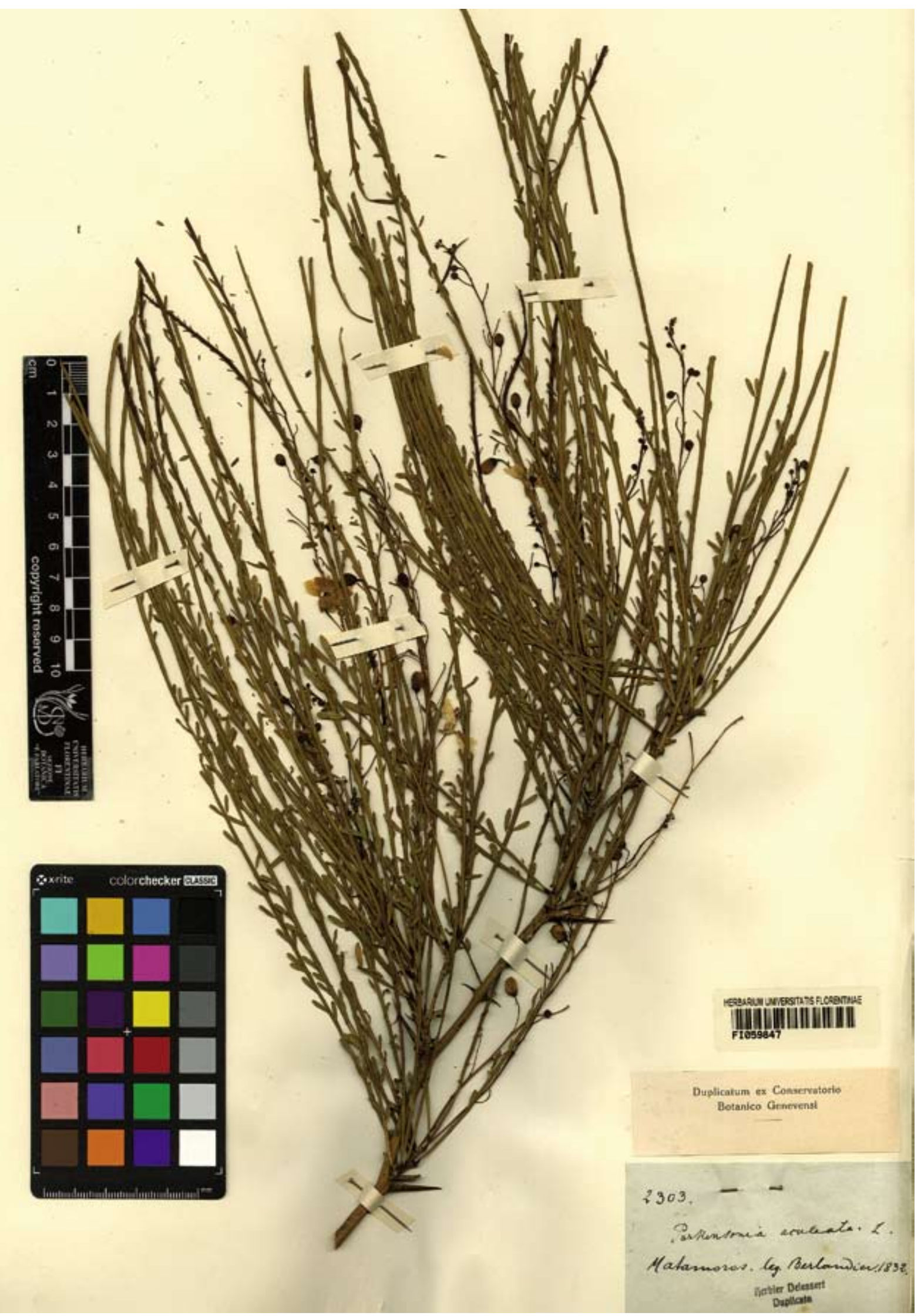

Fig. 3 - Parkinsonia aculeata L. (Fabaceae). American species collected in 1832 at Matamoros (Chihuahua, Mexico) by the FrancoMexican botanist J.-L. Berlandier (courtesy of the Department of Botany in the Natural History Museum of the University of Florence). / Parkinsonia aculeata L. (Fabaceae). Specie americana raccolta nel 1832 presso Matamoros (Chihuahua, Messico) dal botanico francomessicano J.-L. Berlandier (per gentile concessione del Dipartimento di Botanica del Museo di Storia Naturale dell'Università di Firenze). 
Cyperus difformis Linn. Dans les risières et dans les lieux marecageux de l'Italie oû il fleurit aus mois de Sept. et d'Octobre, vivace.

Plante très repandue dans les pays tropicaux et temperés du globe. En Italie, en Sicile, en Dalmatie, en Grèce, en Egypte, au Cap de bonne esperance, dans les Indes Orientales, dans les iles Philippines, dans l'ile de Java, en Chine, à la Nouvelle Hollande etc.

Cyperus mucronatus Rottb Parl. Fl[ora] ital[iana]. cyperus lateralis Forskahl

Dans les lieux humides et marecageux de l'ile de Pantellaria oû elle fleurit en Mai et Juin, vivace.

-Ce trouve encore en Egypte, dans la Thébaide, dans l'Arabie petrée et dans les Indes Orientales

Cyperus rotundus Linn. Très commune dans les champs, dans les lieux sabloneux long les rivières de la partie moyenne et meridionale de l'Italie, et de la Sicile et des petites iles d'Ischia, Saline, Stromboli, Panaria, Lipari, Favignana et Pantellaria.

-Très repandue sur la surface du globe oû elle aime à preference les pays chauds et tropicaux. Se trouve dans le midi de l'Europe en France, en Italie, en Sicile et les iles adjacentes, en Dalmatie, en Grèce, en Macedonie, en Bythinie,en Afrique en Algérie, dans les iles Canaries, en Égypte au Kordofan, au Sennaar, au Sénégal, en Guinée, au Cap de bonne Esperance, dans les iles de France [Mauritius] et de Bourbon [La Réunion], en Asie en Arabie, dans les Indes Orientales, dans l'ile de Cylon, en Chine, en Océanie, dans les Philippines, dans les Mariannes, dans l'ile de Java, dans la Nouvelle Hollande, dans l'Amerique boreale et meridionale.

Cladium Mariscus R. Brown Dans les lieux marécageux, dans les lacs de l'Italie et des iles de Corse, de Sardaigne et de Sicile: fl[eurit] Mai-Juin. vivace

Se trouve en Europe de la Suède et des iles Britanniques jusque en Sicile et en Sardaigne. En Afrique dans les iles Canaries, en Algérie et au Cap de Bonne Ésperance, dans les iles de France et de Madagascar, en Asie sur les bords de la mer Caspienne, en Sibérie. En Amerique dans la Jamaique, en Océanie à la Nouvelle Hollande, dans les iles Sandwich.

Eleocharis atropurpurea b. minor Kunth! Sur les bords du Tessein Ticino près de Pavie. pl[ante] annuelle

Se trouve en Europe sur les bords du lac de Genève près de Lausanne en Afrique sur les bords du Sénégal. L'éspece dans les Indes Orientales.

Fimbristylis annua Roem? e Schult. Dans les lieux humides et marécageux et dans les risières de l'Italie septentrionale et centrale. Fl[eurit] Julliet-Aout, annuelle.

Dans l'Amerique tropicale. Dans l'ile de Sara [Khara Zira] sur la mer Caspienne après Kunth et à Smirne après Scheuchzer qui la reçu par Sherard

Fimbristylis dichotoma Vahl.

Dans les lieux humides et marécageux, le long des rivières et sur les bords des lacs de l'Italie, oû elle est plus rare dans le nord et devient plus commune dans la côte occidentale de Nice par l'Italie centrale et meridionale jusque en Calabre. Elle croit aussi en Sicile entre Catane et Syracuse. Fl[eurit] Aout-Sept. annuelle

Dans la partie méridionale de l'Europe près les bords de la Mediterranée et de l'Adriatique dans le midi de l'Éspagne et en Italie et Sicile. Se trouve aussi dans les iles Canaries, en Algérie, en Egypte, en Nubie en Kordofan, et en Asie dans les Indes Orientales.

Fimbristylis squarrosa Vahl. dans les lieux humides et marécageux de la Toscane près de Florence à Poggio a Cajano, près de Pise et de Lucques. Fleurit de Juilllet à Septembre.-Se trouve aussi dans l'Amerique tropicale, dans l'Afrique tropicale et aux Indes orientales.

Scirpus supinus Linn. dans les lieux humides et marécageux de l'Italie du nord. Fl[eurit] Juill. Oct., annuelle Très repandue sur la surface du globe. En Europe, en Afrique, dans les Indes Orientales et à la Nouvelle Hollande.

Scirpus mucronatus Linn. Dans les lieux marécageux et à préference dans les risières de l'Italie et de la Sicile. Juin - Aout. annuelle.

Scirpus maritimus Linn. Plante commune dans les lieux humides et marécageux, le long des rivières, sur les bords des lacs de la peninsule et des iles italiennes. MaiJuill. vivace, Se trove très repandue dans les deux hemisphères en Europe depuis l'Ecosse et la Suède temperée jusque au Caucase, en Siberie, dans les Indes Orientales, dans la Nouvelle Hollande, dans les iles de Sandwich, en Afrique depuis les iles Canaries et l'Algérie en Egypte, au Sénégal, au Cap de Bonne Ésperance, dans l'Amerique boréale.

Scirpus Michelianus Linn. Dans les lieux humides et marécageux de l'Italie oû il se fait plus rare dans la partie centrale et meridionale. Fl[eurit] Juin-Juill. ann.

En Europe, de la Silesie par l'Allemagne, les iles de la Dalmatie et l'Italie jusque à Naples et en France. En Asie sur les bords de la mer caspienne, en Perse, dans les Indes Orientales, en Afrique en Algérie après Desfontaines et en Senegambie.

Graminées communes aux deux hemisphéres, ou à l'ancien et le nouveau continent, ou aux pays tropicaux de l'Italie

+ Ehrharta panicea Smith, Parl[atore] Flora italia$n a$, vol. I. p.56. Se trouve près Naples à Portici, oû je la crois échappé du jardin royale, et au Cap de Bonne Esperance

Anthoxanthum odoratum Linn. Parl. fl. ital. I. p.58. Se trouve en Italie, en Europe très abondamment et dans l'Amerique du Nord.

Holcus lanatus Linn. En Italie et dans l'Europe moyenne et meridionale et dans l'Afrique boreale, aux iles Canaries et dans l'Amerique du Nord Parl. fl.it I p.63.

Holcus mollis Linn. Parl. fl. it. I. p.64. Dans l'Europe moyenne et meridionale et dans l'Amerique du Nord et Caucase, en Siberie

+ Phalaris minor Retz Dans les pays de la Mediterranée en Europe, en Berbérie, dans les iles Canaries, en Egypte, en Arabie, au Cap de Bonne Esperance Parl. fl.it I p.70.

Phalaris arundinacea Linn. Parl. fl. it. I. p.76. Dans l'Europe moyenne et meridionale, dans le Caucase, en Siberie et dans l'Amerique du Nord

Phleum pratense Linn. Parl fl. it. I, p. 80 En Europe, au Caucase, en Sibérie, dans l'Amerique du Nord 


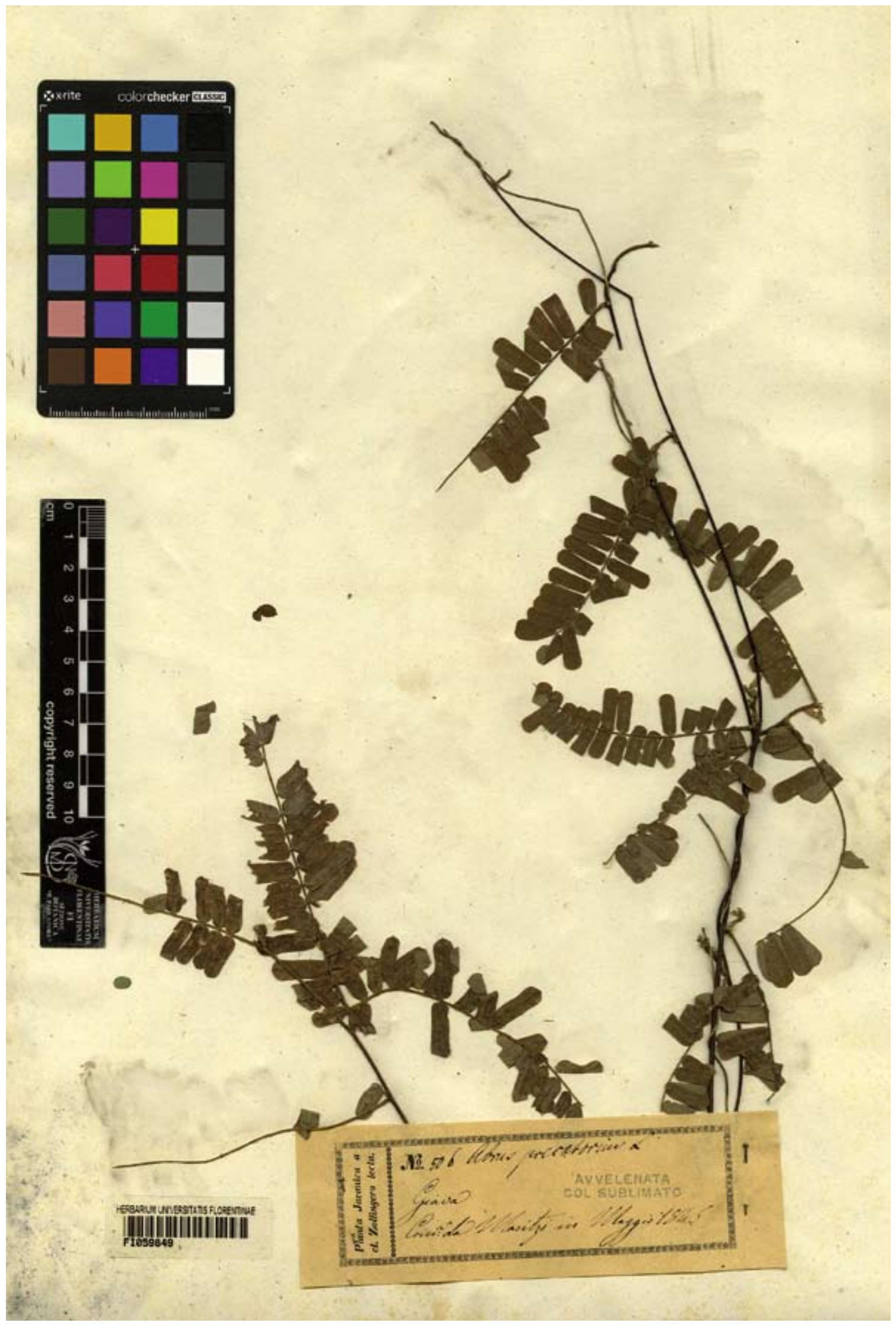

Fig. 4 - Abrus precatorius L. (Fabaceae). Plant with colored seeds for tribal use (necklaces, rosaries), collected in Java in 1845 by the Swiss naturalist Alexander Moritzi (courtesy of the Department of Botany in the Natural History Museum of the University of Florence). / Abrus precatorius L. (Fabaceae). Pianta con semi colorati d'uso tribale (collane, rosari), raccolta a Giava nel 1845 dal naturalista svizzero Alexander Moritzi (per gentile concessione del Dipartimento di Botanica del Museo di Storia Naturale dell'Università di Firenze). 
Beckmannia erucaeformis Host. Parl, fl. it. I. p. 89 En Italie, en Dalmatie, en Orient, en Siberie, dans l'Amerique du Nord

Alopecurus pratensis Linn. Parl. fl. it.. I. p. 96. Dans l'Europe moyenne et meridionale, dans l'Afrique boreale, au Caucase, en Perse, en Siberie et dans l'Amerique du Nord

Alopecurus geniculatus Linn. parl. fl. it. I. p.99 - En Europe, dans l'Afrique boreale, en Sibérie, au Japon, dans l'Amerique boréale.

+ Pennisetum cenchroides Rich. Près de Palerme et dans l'ile de Lipari, sur les côtes de l'Afrique, en Barbarie, en Arabie et au Cap de Bonne Esperance Parl. fl. it. I p.108.

Setaria verticillata

P[alis.] [de] B[eauv.]

Parl. fl. it. I. p. 110

Setaria glauca $\mathrm{P}$ [alis.]

[de] B[eauv.]

Parl. fl. it. I p. 112

Setaria viridis P[alis.] [de] B[eauv.] Parl. fl. it.p.111 très repandue dans l'hemisphère boreale

Panicum crus galli Linn. Parl fl. it.I. p.115 presque cosmopolite

Panicum colonum Linn.Parl. fl. it. I p.117 très répendue depuis la region mediterranéenne jusque dans les tropiques, en Europe, en Asie, en Afrique, en Océanie, en Amerique

Panicum capillare Linn. parl. fl. it. I p.121 En Europe près Nice, Vienne, en Belgique et à Toulon et dans l'Amerique boreale

Digitaria sanguinalis Scop. Parl. fl. it. I p.125 Plante cosmopolite

Tragus racemosus Hall. Parl. fl. it. I p.129 Repandue dans les pays temperés meridionaux et dans et dans les tropicaux du nouveau et de l'ancien monde

+ Imperata cylindrica Palis. de Beauv. Parl. fl. it. I p.137. Se trouve dans le midi de l'Europe, en Egypte, en Arabie, au Cap de Bonne Esperance, dans les Indes Orientales, dans l'ile de Java, à la Nouvelle Hollande, au Chili, etc.

+ Andropogon ischaemum Linn. Parl. fl. it. I p.139 dans le midi de l'Europe, au Caucase, au Cap de Bonne Ésperance et dans les Indes Orientales

+ Andropogon hirtum Linn. Parl. fl. it. I p.139. Dans le midi de l'Europe, en Arabie, en Egypte, dans les iles Canaries et du Cap vert, au Cap de Bonne Ésperance etc.

+ Stipa tortilis Desf. Parl fl. it. I p.168. Dans le midi de l'Europe, dans les iles Canaries, en Algérie, en Egypte, au Cap de Bonne Ésperance, en Arabie, en Perse.

Chaetaria adscensionis Palis. de Beauv. Parl. fl. it. I p.172 Près Palerme oû je l'ai decouvert le premier, près de Malaga en Éspagne, dans l'Afrique boreale, dans les iles Canaries, du Cap Vert, dans l'Arabie petrée, dans l'ile de l'Ascension et au Bresil.

Sporobolus pungens Kunth Parl. fl. it. I p.196. Dans le midi de l'Europe, en Orient, dans l'Afrique boréale, à la Guadaloupe.

Polypogon monspeliense Desf. Parl. fl. it. I p.198. Très répendue dans notre hémisphère, dans les iles britanniques jusque à l'Afrique boreale, en Egypte et en Arabie. Se trove dans l'autre hémisphère à Montevideo. [in Humboldt's handwriting] Géographie des Plantes

+ Polypogon maritimum Willd. Parl. fl. it. I. p.200 Sur les bords de la Méditerranée et de l'Adriatique au midi de l'Europe en Egypte, aux iles Canaries, au Cap de Bonne Ésperance, en Siberie et dans les Indes Orientales.

Ammophila arundinacea Host. Parl. fl. it. I. p.214 En Europe sur les bords de la mer Baltique, de la mer Mediterranée et de l'Adriatique. Elle croit aussi dans l'Amerique du nord.

Phragmites communis Trin. Parl. fl. it. I. p.220 Plante cosmopolite

Cynodon dactylon Pers. Parl. fl. it. p.223 Plante cosmopolite

Dactyloctenium aegyptium Willd. Au midi de l'Europe en Calabre et en Sicile, en Egypte, dans le Fazoql [in the northern part of Sudan], en Arabie, dans les Indes Orientales, dans les Moluques, en Amerique Parl. fl. it. I. p.225

Dinebra arabica Palis. de Beauv. Parl. fl. it. I. p.227. En Italie sur les bords de l'Adriatique près d'Ascoli, de Giulianova, e Pescara, en Egypte, dans le Fazoql, en Senengambie, en Arabie, dans les Indes Orientales.

Catabrosa aquatica Palis. Parl. fl. it.- I. p.238 En Europe, dans le Caucase, en Sibérie, dans l'Amerique du Nord.

+ Avenella flexuosa Parl. fl. it. I. p.246. En Europe depuis la Laponie et la Suede jusque en Sicile, dans le Caucase, en Sbérie, dans l'Amerique du Nord; elle se trouve aussi dans l'autre hemisphère dans les iles de Falkland, d'oû je l'ai reçu par Mr. Hooker fils.

+ Aira caryophyllea Linn. Parl. fl. it. I. p.251 En Europe, en Asie et dans l'autre hemisfère au Chili et au Cap de Bonne Ésperance.

Trisetum subspicatum Palis. de Beauv. Parl. fl. it. I. p.258. Dans les climats polaires de notre hémisfère en Laponie, Suède, dans l'Amerique arctique elle croit aussi dans les Alpes et dans les Pyrenées. Mr. Hooker fils le porta de l'autre hemisfère, mais les échantillons qu'il m'a donné sous ce nom sont bien differents de l'éspece arctique et ils appartiennent probablement à une éspece nouvelle.

Trisetum flavescens Palis. de Beauv. Parl. fl. it. I. p.261 Dans les pays temperés de notre hemisfère en Europe, en Asie, en Amerique. se trouve de preference dans le midi.

+ Danthonia provincialis Dec. Parl. fl. it. I. p.296. Dans la Provence, dans le Dauphiné, en Italie, en Carinthie, en Carniolie et dans la Nouvelle Hollande.

+ Cynosurus echinatus Linn. 336 Dans les pays temperés de deux hemisfères. Se trouve a préference dans le midi de l'Europe, en Orient, dans le Caucase, dans l'Afrique boreale et à Montevideo.

Poa annua Linn. Parl. fl. it. I. p.348 Plante cosmopolite

Poa compressa Linn. Parl. fl. it. Parl. p.350 Très rependue dans les pays temperés de l'hemisfère boreale, en Europe, en Asie et en Amerique

Poa pratensis Linn. Parl.

fl. it. I. p.352

Poa trivialis Linn. Parl.

fl. it. I. p.354

Poa nemoralis Linn. Parl. fl. it. I. p.358
Très repandues

dans les pays

temperés de

l'hemisfère boreale 


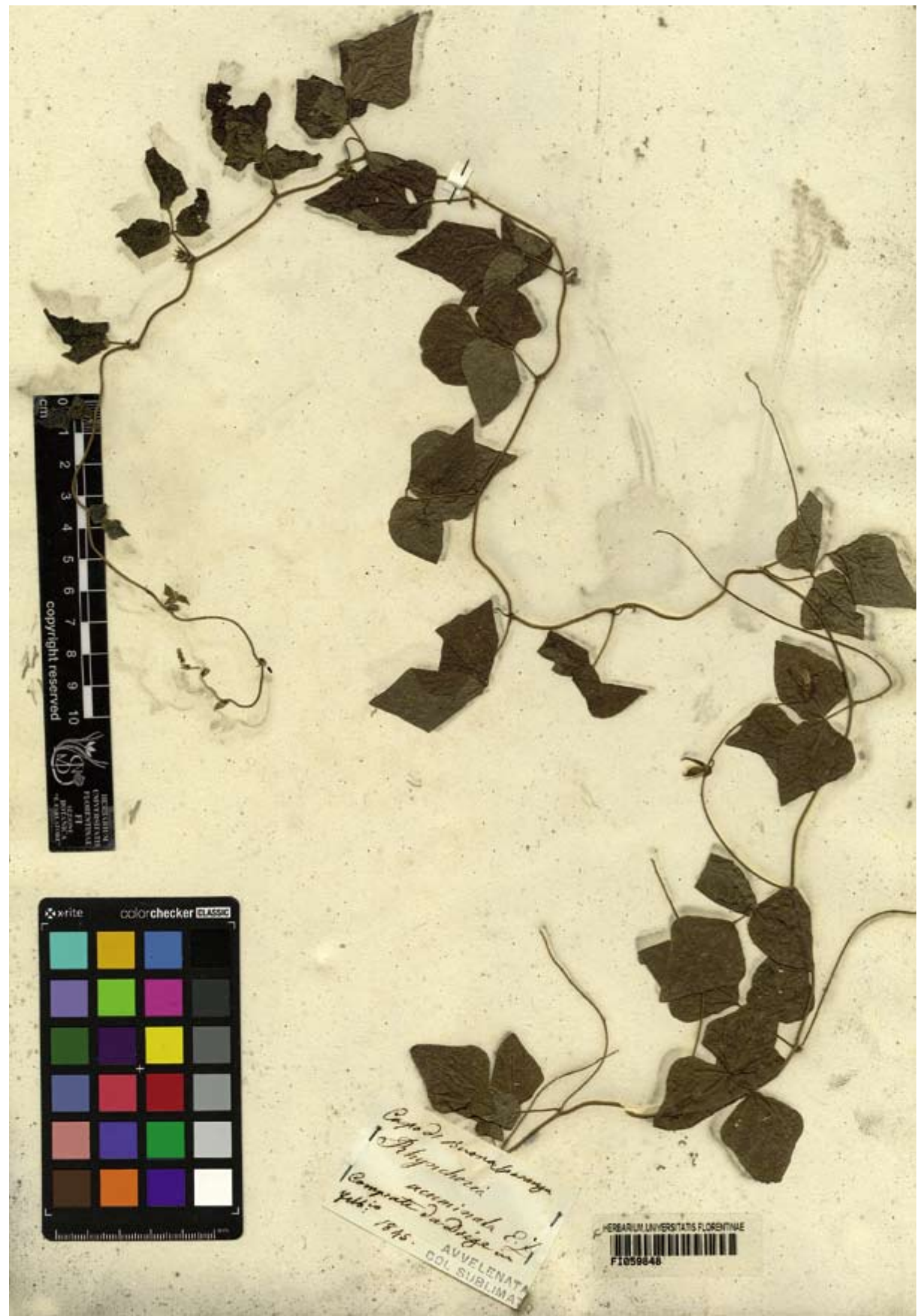

Fig. 5 - Rhynchosia caribaea (Jacq.) DC. (= R. acuminata Eckl. \& Zeyh., nom. superfl.). (Fabaceae). Leguminous plant of centralsouthern Africa received by Parlatore from the Cape of Good Hope (courtesy of the Department of Botany in the Natural History Museum of the University of Florence). / Rhynchosia caribaea (Jacq.) DC. (= R. acuminata Eck1. \& Zeyh., nom. superfl.). (Fabaceae). Leguminosa dell'Africa centro-meridionale pervenuta a Parlatore dal Capo di Buona Speranza (per gentile concessione del Dipartimento di Botanica del Museo di Storia Naturale dell’Università di Firenze). 
Glyceria aquatica Wahlb. Parl. fl. it. I p.372 Très repandue dans l'hemisphère boreale

Eragrostis megastachya Link. Parl. fl. it. I. p.380. dans le midi de l'Europe, dans l'Afrique boreale, dans l'Egypte, le Fazogl et l'Amerique.

+ Briza minor Linn. Parl. fl. it. p.382 Dans l'Europe meridionale, dans l'Afrique boreale, en Perse, au Cap de Bonne Ésperance, dans le Bresil, le Perou.

+ Briza maxima Linn. parl. fl. it. p.386 Dans le midi de l'Europe, en Orient, dans l'Afrique boreale, dans les iles Canaries, au Cap de Bonne Ésperance, dans les Indes Orientales.

+ Serrafalcus mollis Parl. fl. it. I. p.395 Dans l'Europe moyenne et meridionale, au Caucase, dans l'Afrique boreale et au Chili

+ Vulpia myuros Gmel. Parl. fl. it. I. p.418 Dans l'Europe moyenne et meridionale, en Algerie, dans l'Asie mineure, au Cap de Bonne Ésperance, en Mexique.

Dactylis glomerata Linn. Parl. fl it. I. p.458. En Europe, en Siberie et dans l'Amerique du Nord

Agropyron junceum Palis. Parl. fl. it. I. p.502 Sur les bords de la mer dans l'Europe moyenne et meridionale, au Caucase, en Siberie, en Algerie, en Egypte et dans l'Amerique du Nord

Hordeum murinum Linn. Parl. fl. it. I. p.520. En Europe, en Orient, en Arabie, dans l'Afrique boreale er dans l'Amerique du Nord

Hordeum secalinum Schreb. Parl. fl. it. I. p.521 dans l'Europe moyenne et meridionale en Orient et dans l'Amerique du Nord

Hordeum maritimum With. Parl. fl. it.I p.522 Très repandue en Europe, en Asie et en Amerique

Lolium perenne Linn. Parl. fl. it. I p.529. Très commune en Europe, au Caucase, dans l'Amerique du Nord.

+ Lepturus cylindricus Trin, Parl. fl. it. I p.539. Dans le midi de l'Europe, dans les iles Canaries, au Cap de Bonne Ésperance.

[in Humboldt's handwriting] Parlatore Mai 1851

[Humboldt's handwritten notes on the letter itself]

[top]

Ischia

Pteris Longifolia von amer[i]ck[a]ner Trop[en] bis

[centre]

Dureau de la Malle [A.] (Climatologie comparée de l'Italie et de l'Andalousie anciennes et modernes p.2 1849) de nouveau tire l'attention sur l'obs[ervation] de Tenore qui a trouvé a Ischia pres des [referral sign]

[bottom]

[referral sign] fumarolles de Caciotti Pteris longifolia et Cyperus polystachyus ${ }^{23}$

Parlatore croit que l'Italie du sommet des Alpes - Sicile, de 6000 à 7000 Phanerog[ames] parmis le[s]q[uelles] 700 ? Graminées

Frage [wieder]

[Humboldt's handwritten notes on the sheet glued to the letter]

Parlatore.
Musa parad[isiaca] se cultive im Freien und tragt Fruchte bei Catania, Canna indica ist jetz verwildert bei Sicilien, in San Cosimano bei Mililli.

Flora Ital 1850 I p.32.

Hochste Phaner[ogame] [die] Saussure [auf] Alpen [gesehen]. Silene acaulis Montblanc 1780 t[oises]

Aretia helvetica u[nd] A. imbricata. 1763 t[oises] Col de Geant

Parlat. auf Etna hochste phanerog[ames] Rumex aetnensis, Senecio aetnensis, Robertia taraxacoides bei Casa degli Inglesi 9028 par[iser] $\mathrm{F}$ [üsse] = 1505 t[oises] [auf] Montblanc $8^{\circ}$ lat[itude] Ursach bloss [jah] hoher am Etna alles lava $\mathrm{u}[\mathrm{nd}]$ scorie

Flora it[aliana]. Pref[azione] p.11

[Humboldt's notes on the envelope containing the letter]

Parlatore

Geographie botanique

demander

erica

[...] perennis [wir] [west].

Papyrus

Quercus

wilde Kartoffeln

[...] Enna

$[\ldots]$ am Fremdesten

Pantellaria

Polygon[um] aviculare

Hord[eum] murinum

Alsine media

Plantago

For the letter, the notes on the sheet glued to the letter, and the list of plants enclosed:

Staatsbibliothek zu Berlin - Preußischer Kulturbesitz Nachl. Alexander von Humboldt, gr. Kasten 13, Nr. 24

$<$ https://digital.staatsbibliothek-berlin.de/werkansicht ?PPN=PPN838243401\&PHYSID=PHYS_0001 $>$

For the envelope:

Staatsbibliothek zu Berlin - Preußischer Kulturbesitz Nachl. Alexander von Humboldt, gr. Kasten 12, Nr. 90.91, B1. 9

$<$ https://digital.staatsbibliothek-berlin.de/werkansicht ?PPN=PPN829064702\&PHYSID=PHYS_0001 $>$

\section{Letter 3}

[from Humboldt to Parlatore]

J'ai traité dans mon ouvrage Examen critique de l'Histoire et de la Géographie du Nouveau Continent (1837) Tome II, p. 76-78 des plantes communes aux côtes opposées de l'Afrique et de l'Amerique méridionale: j'ai indiquée mes sources. Faites moi l'amitié d'examiner et d'employer votre hereuse sagacité.

Y-a-t-il dans les plantes Magellaniques et du Chili austral quelque chose d'Européen ou du Canada?

Croyez vous à 2-3 phanérogames qui seraient partout même sous les tropiques: croyez vous à l'origine de l'Erigeron canadense ${ }^{24}$ ? 


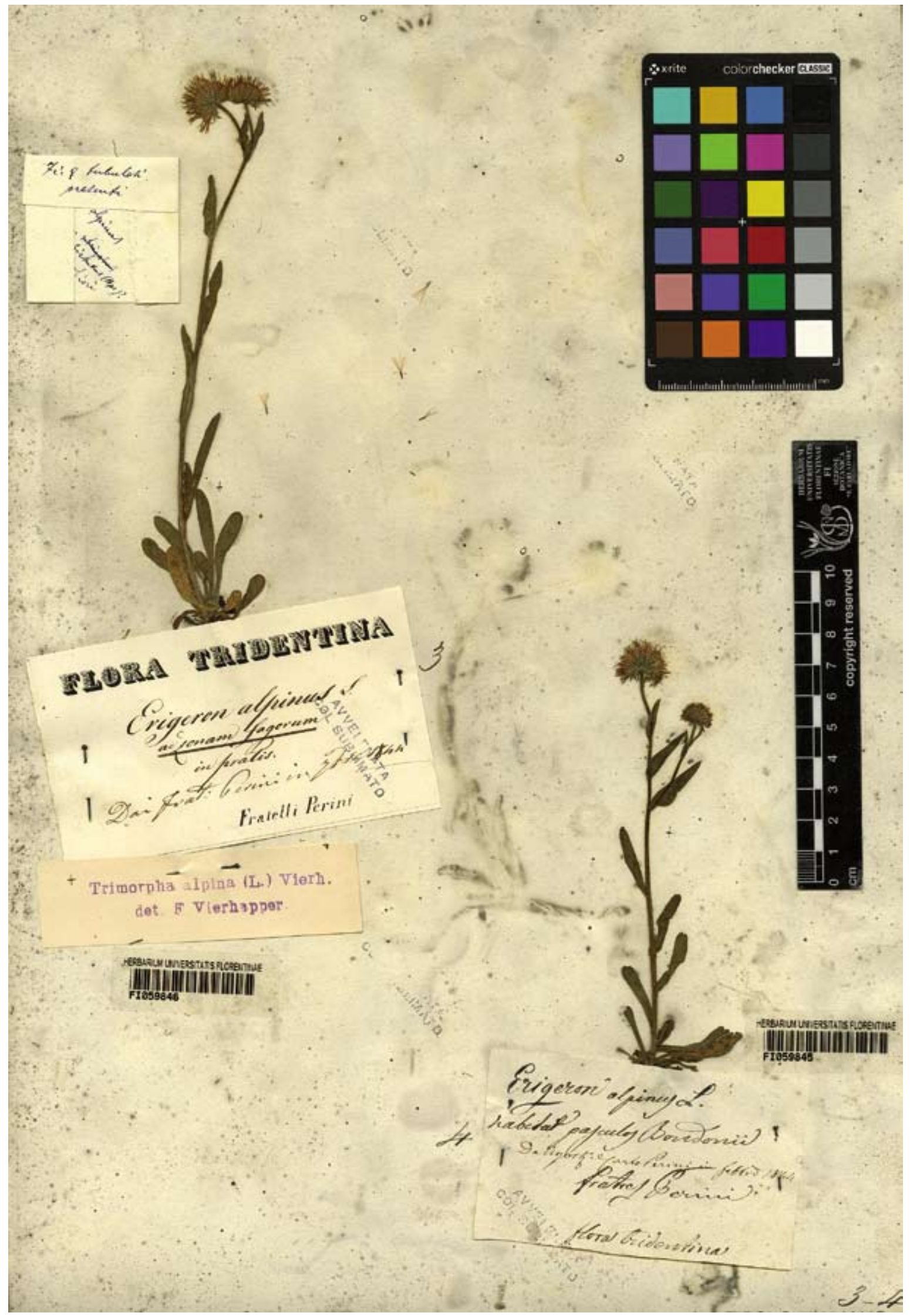

Fig. 6 - Erigeron alpinus L. (Asteraceae). Italian collection of brothers A. and C. Perini made in Trentino, plant received by Parlatore in September 1844 (courtesy of the Department of Botany in the Natural History Museum of the University of Florence). / Erigeron alpinus L. (Asteraceae). Raccolta italiana dei fratelli A. e C. Perini effettuata in Trentino, pianta ricevuta da Parlatore nel Settembre del 1844 (per gentile concessione del Dipartimento di Botanica del Museo di Storia Naturale dell’Università di Firenze). 
Daignez jeter les yeux sur la distribution des Familles des Plantes dans le second volume de mes Tableaux de la Nature (1851) traduction de Mr. Galuski Tome II, pp. $37-$ 243. Rareté des Ombellifères et Crucifères sous les tropiques dans les plaines, non sur les montagnes, Humboldt, De distributione geographica Plantarum secundum coeli temperiem et altitudinem montium Prolegomena $(1817)^{25}$, p. 38; dans l'Amérique septentr[ionale]. il y a sur 2900 plantes phanérog[ames] 385 espèces européennes, p. 53; enumération des plantes européennes dans l'Emispher austral, p. 57; Cypéracées et Graminées de la zone tempéré boreale que nous avons trovées sous les tropiques, pas une seule Joncacée, p. 64; pas de phanérogame dycothiledone long des côtes, p. 62 et 65 ; mêmes genres mais d'autres espèces, p. 62; sur les côtes Rhiz[ophora] Mangle, Avicennia tomentosa, Sesuvium portulacastrum, presque sur toutes les côtes des régions tropicales de la terre p. 61. Jetez de grace les yeux sur mon Tableau comparatif des zones p. 88-165 Tout cela ne se trove pas dans les Nova Genera et species plantarum ${ }^{26}$.
Affectueux hommages
A. v. Humboldt
ce 20 Mai 1851

Biblioteca Comunale di Palermo, Fondo Parlatore, 5 Qq D $16(22 / 2)$

\section{Letter 4}

[from Humboldt to Parlatore]

Je ne voudrais pas laisser partir le savant et aimable Chevalier de Réumont ${ }^{27}$ pour Florence sans Vous donner, mon respectable ami, un signe de vie et de reconnaissance pour les moments instructifs que Vous m'avez accordés lors de vôtre passage à Berlin. J'ai appris avec un vif plaisir que le Roi Oscar a fait honneur à ma recommandation et que Vous avez reussit dans vôtre grande et noble entreprise. Des fréquentes et longues absences du $\operatorname{Roi}^{28}$ ont entravé l'accomplissement de mon désir le plus vif, de Vous annoncer combien Vos éminents travaux de botanique et de physique comme la grande et noble entreprise d'une Géographie végétale de l'Europe ont été appreciés. Le Roi a reçu avec un bienveillant intérêt Vos recherches sur l'influence du climat alpin ${ }^{29}$ et le Ier volume de la Flora Italiana. Il vient de Vous nommer Chevalier de son Ordre Royale de l'Aigle Rouge; troisième Classe. Vous aurez une lettre autographe très flatteuse du Roi qui Vous confirmera cette nomination ${ }^{30}$. Monsieur! Je vais ajouter l'observation peu philosophique que la 3eme Classe de l'Ordre est celle que l'on a donné aux hommes les plus célèbres en France et en Russie tandisque les savants indigènes, voire les Ministres d'état (que chez nous on met pas au galères comme en certains pays volcaniques ${ }^{31}$ ) ne déboutent que par la 4me. Je viens d'annoncer Votre nomination à S. A. Imp. et Royale le Grand Duc en Lui répétant tout ce que je sents pour Vous. J'espère que lorsque Vous en aurez le loisir Vous me donnerez quelques notices de Géogr[aphie] botanique relatives à des questions qui me tiennent à coeur. Excusez je Vous supplie la hâte de ces lignes et veuillez bien offrir mes affectueux homma- ges à l'illustre Chev. Antinori ${ }^{32}$, comme à l'ingénieur Mr. Nobili ${ }^{33}$ !
à Sans Souci le 12 Nov. 1851
A. v. Humboldt

Biblioteca Comunale di Palermo, Fondo Parlatore, 5 Qq D $16(22 / 4)$

\section{Letter 5 and enclosure}

[From Parlatore to Humboldt]

\section{Excellence!}

Je regrette vivement de n'avoir pas pu vous envoyer plus tôt notices relatives à quelques points de géographie botanique, que je vous avais promises dans ma lettre du mois de Janvier à cause de la necessité oû je me suis trouvé de rester chez moi jusqu'au mois de Mars sans puvoir me rendre à l'herbier pour y faire les recherches nécessaires. Je vous prie de vouloir m'excuser de ce retard qui m'a été bien penible puisque il s'agissait de Vous, qui outre le mérite éminent qui vous distingue avez été pour moi particulierment un vrai bienfaiteur. J'éspère que ce que je transmets sur les points en question de géographie botanique ne trompera tout à fait votre attente: je me suis borné à vous écrire ce dont j'étais sur grâce à mes études.

Mr. Basevi ${ }^{34}$, jeune florentin très instruit et qui est très hereux de pouvoir par cette occasion avoir l'honneur de faire la connaissance personelle d'un homme qui a rempli de sa gloire le monde entier, vous portera avec cette lettre les dernièrs cahiers du Journal botanique italien ${ }^{35}$ et la première partie du second volume de ma Flora italiana qui vient de paraitre et qui comprend toute les cyperacées italiennes. J'ai taché dans cette famille de detailler d'une manière plus exacte les limites géographiques de chaque plante en Europe etc, et de préparer ainsi mon travail sur la flore d'Europe ${ }^{36}$ que j'éspère publier plus tard après la Flora italiana. Je travaille maintenant au reste des monocotylés italiennes que je publierai plus tard ${ }^{37}$, mais je ne puis pas aller trop vite, car il faut bien du temps, comme vous le savez mieux que moi, pour travailler cosciencieusement. J'ai déjà beucoup avancé mon manuscit de la partie déscriptive de mon voyage en Laponie et j'éspère en commencer l'impression dans l'automne prochain pour m'occuper ensuite de la partie botanique de ce voyage ${ }^{38}$, dont je rassemble maintenant les materiaux.

Dans le paquet que je vous envoye vous trouverez aussi un petit memoire de moi sur les jardins botaniques; et c'est ce memoire dont j'eu l'honneur de vous parler à Berlin et dans le quel j'ai proposé de destiner une partie de ces jardins à des tableaux de géographie botanique (Parlatore F., 1846).

Encouragé par la bonté genereuse de Sa Majesté le Roi de Prusse à mon égard j'ose Lui adresser avec une lettre la première partie du second volume de la flore italienne et les cahiers du journal botanique dont il est question plus haut: je vous prie de vouloir bien avoir la bonté de les présenter en mon nom à Sa Majesté et de lui témoigner de ma part mieux que je ne puis par écrit toute ma réconnaisance pour les marques de la généreuse protection qu'il m'a accordée ${ }^{39}$. 


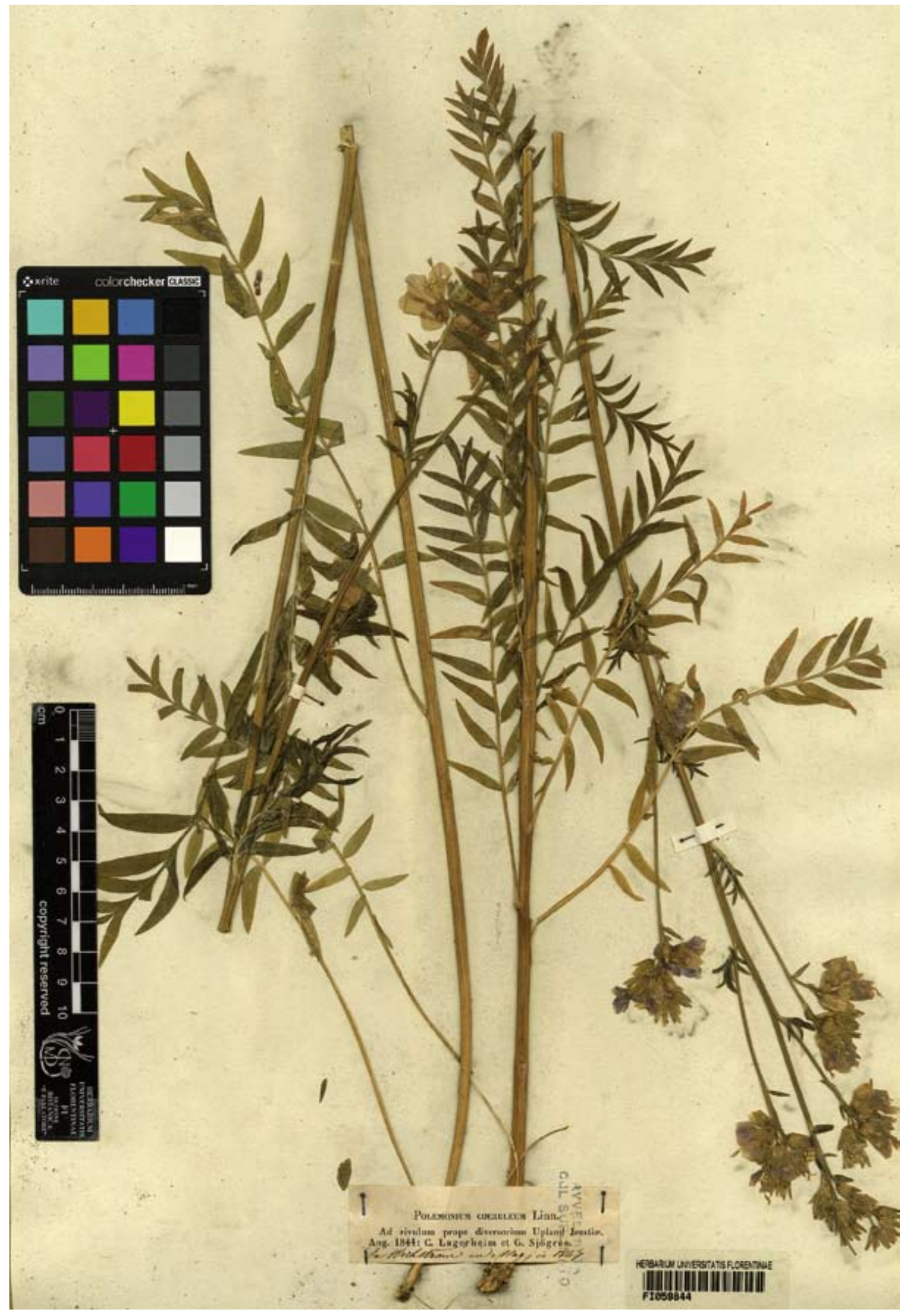

Fig. 7 - Polemonium caeruleum L. (Polemoniaceae). Arctic-Alpine plant collected in Sweden (Jämtland County) by J. Emanuel Wikström in 1844 (courtesy of the Department of Botany in the Natural History Museum of the University of Florence). / Polemonium caeruleum L. (Polemoniaceae). Pianta artico-alpina (raccolta in Svezia (Contea di Jämtland) da J. Emanuel Wikström nel 1844 (per gentile concessione del Dipartimento di Botanica del Museo di Storia Naturale dell’Università di Firenze). 
J'éspère voir paraitre bientôt le dérnier volume de votre Cosmos, je l'attends comme tout le monde, avec une grande impatience; vous devez etre bien content de conduire à fin un ouvrage dont heureusement la grandeur du sujet est cette fois en harmonie avec le genie de l'auteur.

Ma santé s'est beaucoup amelioré dans ces derniers temps; je vais partir dans peu de jours pour les bains de Lucques et j'éspère en retourner tout à fait guéri.

Je prie Dieu de vous accorder des longues et heureuses années pour le bien des sciences, de la societé, des amis et de celui qui a l'honneur d'étre avec le plus profond respect et la plus haute considération.

de V. E.

Le Baron Alexandre de Humboldt

etc etc le très humble et devoué serviteur

Florence ce 23 Juin $1852 \quad$ Philippe Parlatore

Le memoir sur les jardins se trouve inseré dans le n. 9 du journal botanique

\section{[Enclosure] [from Parlatore to Humboldt]}

Florence le 23 Juin 1852

J'ai porté mon attention sur les plantes qu'on croit être communes aux côtes opposées de l'Afrique et de l'Amerique et voici les résultats de mes études sur ces plantes.

Il y a un nombre à peu près de 25 à 30 éspèces de plantes qui croissent en même temps sur le côtes du Bresil ou de la Guyane en Amerique et sur les côtes de la Sénegambie, du Congo etc. en Afrique. Dans ce nombre il faut distinguer: 1 . les plantes qui vivent communement sur les bords de la mer dans les pays tropicaux, telles que le Rhyzophora mangle, l'Avicennia tomentosa etc.: 2. les plantes qui se trouvent presque partout dans les lieux cultivés des pays tropicaux, telles que l'Argemone mexicana, la Sauvagesia erecta etc. 3. d'autres qui ont très probablement été introduites par la culture, le Cassia occidentalis, la Guilandina Bonduc, le Parkinsonia aculeata [Fig. 3], l'Abrus precatorius [Fig. 4], le cardiospermum hali, cacabium, etc., et 4 . Enfin les plantes qui semblent être tout à fait indigènes des deux côtes sans qu'on puisse supçonner leur transport d'un continent à l'autre. Ces plantes sont au nombre à peu près de dix, l'Urena americana, Malachra radiata, Walteria americana, pterocarpus lunatus, Rhyncosia Caribaea [Fig. 5], Ximenia americana, Dodonea viscosa, conocarpus erecta, chrysobalanus Icaco, etc. Quelques unes de celles ci ne présentent specifiquement aucune difference dans le deux continents, d'autres au contraire telle que le Walteria americana, Rhyncosia Caribaea etc. présentent quelques particularités de forme qui en ont fait faire des éspèces differentes. On peut de tout cela conclure que le nombre des plantes communes aux deux côtes opposées de l'Amerique et de l'Afrique sur le quel on peut compter d'une manière sure, est très borné; au moins pour ce que j'en sache. Il faut noter que je ne sais trouver dans les plantes monocotylés, exepté les cyperacées et les graminées, aucune plante commune aux deux continents. Le petit nombre de palmiers qui crois- sent sur les côtes occidentales de l'Afrique ne présentent aucune espèce et même aucun genre commun à l'Amerique comme l'à déjà bien noté $\mathrm{Mr} \mathrm{R}$. Brown dans ses remarques botaniques sur les plantes recueilles au Congo par Chr. Smith ${ }^{40}$. Cela confirme ce que vous avez ecrit dans votre Examen critique de l'Histoire de la géographie $d u$ Nouveau Continent ${ }^{41}$, c'est à dire qu'on peut appliquer avec de certains réstrictions au règne vegetal la grande lois reconnue par Buffon dans la disparité de la création animale de l'Amerique et de l'Afrique ${ }^{42}$.

Quant aux plantes magellaniques considerées dans leur rapport avec les plantes du Canada et de l'Europe, je crois qu'il en existe un certain nombre qu'on peut regarder comme tout à fait identiques, quoique je suis bien loin de considerer comme telles toutes les plantes prétendues européennes etc. qui croissernt au detroi de Magellan, aux Iles de Falkland etc. Je considère ainsi comme éspèces bien distinctes la primula magellanica, le Trisetum molle qu'on a cru étre la même chose que notre primula farinosa et notre Trisetum subspicatum; je trouve dans plusieurs éspèces, telles le Sisymbrium Sophia, draba incana, Cardamine hirsuta, epilobium tetragonum, Callitriche verna, lycopodium clavatum etc. des telles modifications dans leur formes que l'on doit au moins y reconnaitre des variétés bien marqueées, mais je crois que le Cerastium arvense et vulgatum, le Pisum maritimum, l'Hyppuris vulgaris, le galium aparine, le Senecio vulgaris, le Taraxacum dens leonis, l'erigeron alpinus [Fig. 6] et uniflorus, le statice armeria, le carex festiva et curta, le phleum alpinum, l'avenella flexuosa, l'aira cariophyllea, etc. sont parfaitement identiques avec les espèces qui croissent dans notre hemisphère. Il est vrai que nous pouvons dans ces plantes magellaniques considerer certaines espèces comme certainement introduites dans les terres antartiques, ainsi le pisum maritimum, le Cerastium, le Senecio, l'aira caryphyllea, mais pour d'autres il parait par le rapport des voyageurs et par la nature des plantes, qu'elles y sont tout à fait indigènes ${ }^{43}$.

Vous me demandez si je croi à l'existence de deux ou trois plantes cosmopolites. il est pour moi difficile de repondre à cette demande; cepandant je vous dirais ce que j'en pense.

Si par plantes cosmopolites on entend des plantes qui croissent naturellment sur toute la surface du globe ou au moins dans les trois differentes zones, torride, temperée et glaciale, de deux hémiphères, je crois que aucune plante ne merite de porter ce nom, si on exepte peut être quelques cryptogames surtout quelques espèces de mousses et d'hepatiques, la funaria hygrometrica, le marchantia polymorpha etc. ${ }^{44}$. Il est facile de trouver des plantes qui croissent tout à fait indigènes dans les climats glaciaux et temperés de notre hémisphère, meme en grande abbondance, ainsi l'anthoxanthum odoratum, l'achillea millefolium etc. repandues presque partout en Europe, du Cap Nord jusque en Sicile, et qui se trovent aussi en Asie et en Amerique; on peut meme trover d'autres plantes qui sont abbondantes dans les climats temperés même froids de notre hemisphère et qui reparaissent dans des climats semblables de l'autre hémisphère, ainsi le Samolus valerandi, le Montia minor, l'eleocharis palustris, l'avenella flexuosa, etc., on peut enfin trouver des plantes qui tel- 


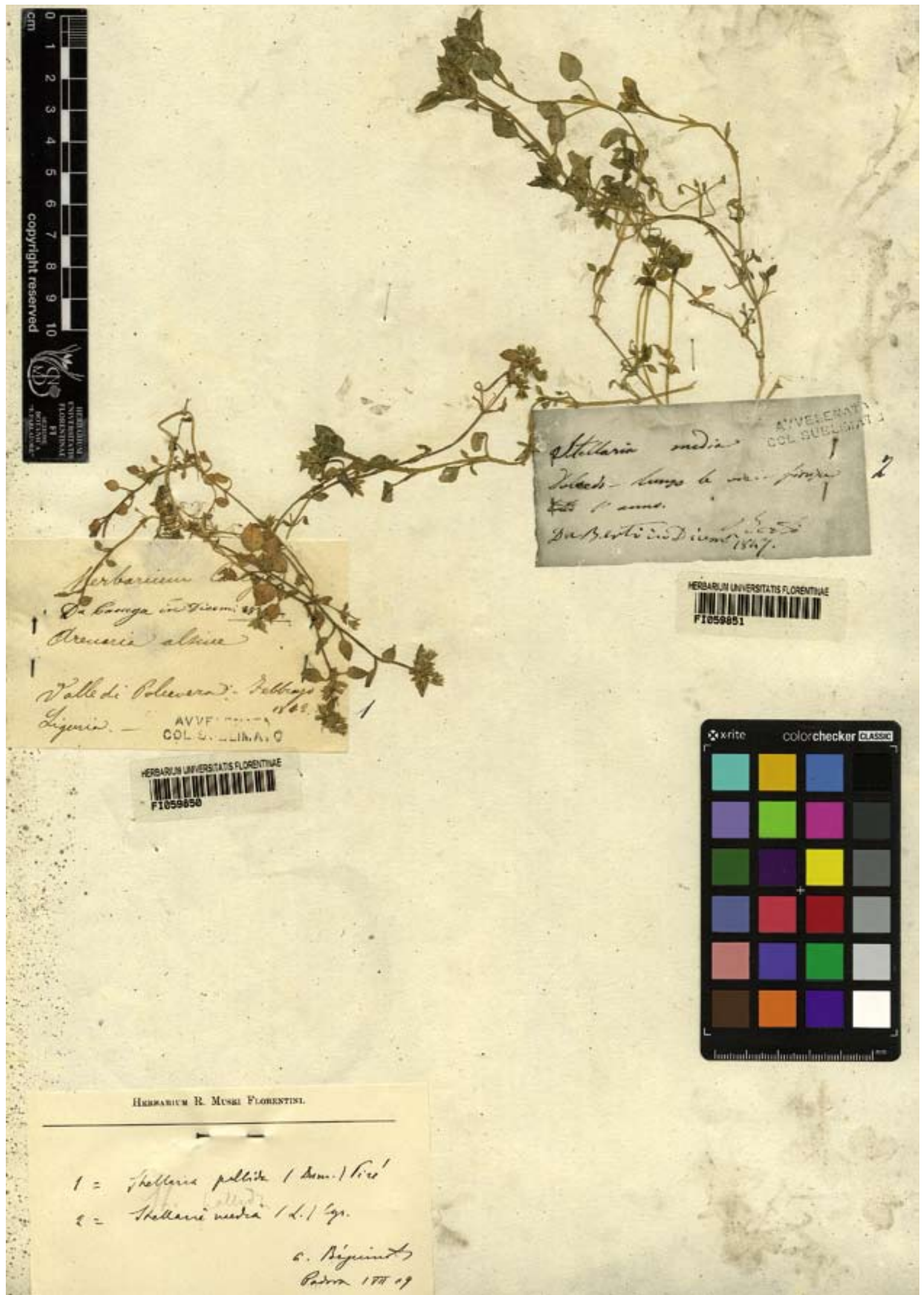

Fig. 8 - Stellaria media L. (Caryophyllaceae). Annual sub-cosmopolitan of Eurasian origin collected in Val Polcevera (Genoa) in 1842 (courtesy of the Department of Botany in the Natural History Museum of the University of Florence). / Stellaria media L. (Caryophyllaceae). Annuale subcosmopolita di origine eurasiatica raccolta in Val Polcevera (Genova) nel 1842 (per gentile concessione del Dipartimento di Botanica del Museo di Storia Naturale dell’Università di Firenze). 
les que le Ranunculus aquatilis habitent les eaux de toute l'Europe depuis la Laponie jusqu'en Sicile, de l'Asie, en Perse, en Syrie etc., de l'Amerique au Mexique etc., de l'Afrique en Abyssinie, au Cap de Bonne Esperance, de l'Océanie à la Nouvelle Hollande etc., mais je ne connais pas de plantes qui soient en même temps indigènes des tropiques et des climats tempérés et glaciaux car généralment les plantes des climats glaciaux s'arrètent aux climats temperés et celles des climats temperés aux tropiques: il y a seulment un petit nombre de plantes qui vivent en même temps dans les pays glaciaux et temperés et d'autres ancore en moindre nombre qui vivent dans les climats tempérés et sous les tropiques; ces dernières sont principalmente des cyperacées, des graminées ou de plantes aquatiques.

On peut ici demander si il y a des plantes que en vivant sur les côtes de la mer dans le pays glaciaux se trouvent à des hauteurs toujours plus considerables dans les pays tempérés et sous les tropiques, en un mot si il y à des plantes communes à toutes les regions alpines de la surface du globe. Quant à cela je crois que il n'existe pas une seule plante qui se trove dans toutes ls régions alpines de la terre, y compris le trisetum subspicatum que quelques botanistes ont cru pouvoir étendre d'une region polaire à l'autre. Pas même quand on voudraut admettre que le trisetum andinum des Andes et le trisetum molle du détroit de Magellan sont la même espèce que le trisetum subspicatum qui croit dans l'Europe, l'Asie et l'Amerique artiques et dans les Alpes, les Pyrenées, les Rocky Mountains etc., nous ne trouvons pas ce trisetum subspicatum dans les Appenins d'Italie, dans le Caucase, dans l'Atlas, dans les Alpes de l'Abyssinie, dans les Alpes de Java etc. qui ont toutes une région alpine. Après mes études sur les differentes régions alpines surtout de l'Europe, j'ai du venir à la conclusion que les plantes de la région alpine des climats glaciaux de l'Europe ne surpassent pas les Appenins des Abruzes et le Caucase, car je trouve que aucune des plantes alpines qui vivent tout près du Cap Nord dans la région alpine des alpes de Norvège, de Laponie et qui se trouvent encore dans nos Alpes, dans les Pyrenées, dans les Appenins et le Caucase ne croit dans l'Atlas, dans les Alpes de l'Abyssinie, etc., quoique la hauteur de ces montagnes admet une région alpine. J'ai pu noter une seule exception pour un petit nombre d'éspèces qui arrivent jusqu'à l'Hymalaya; ce sont le Sibbaldia procumbens, la gentiana tenella, le polemonium caeruleum [Fig. 7], je n'ai pas ces plantes dans mon herbier de l'Hymalaya; par consequence je ne me rend pas garant de ces determinations: c'est un fait à étudier dont je m'occuperais dans la partie botanique de la rélation de mon voyage au Cap Nord de l'Europe ${ }^{45}$.

Il reste à savoir si il y à des éspèces cosmopolites à cause de la facilité qu'elles ont à vivre sur toute la surface du globe une fois que elles y ont été transportés, comme il en est pour certains animaux domestiques.

Ce sont ces espèces qui semblent s'attacher aux pas des hommes et qui paraissent partout où il a un champ cultivé, une maison, un cabane. Je crois que même dans ce sens il n'y a pas de plantes vraiment cosmopolites, quoique le poa annua, la Capsella bursa pastoris, la Sagina procumbens, la Stellaria media [Fig. 8], le daucus carota, soient tres repandu sur le globe.
En générel on peut dire que ces éspèces suivent les mêmes lois que les plantes indigènes, c'est à dire que celles qui vivent dans les pays tropicaux peuvent s'étendre jusqu'aux pays temperées et celles des pays-ci jusqu'aux pays de la zone glaciale: c'est ce que resulte de la distribution géographique des plantes dont il est ici question.

Je ne puis que confirmer la rarété des ombrellifères et des crucifères sous les tropiques dans les plaines, pas sur les montagnes. Pour ce que j'en sache on ne trouve en Afrique et en Asie que cinq ou six éspèces d'ombrellifères et moins de crucifères qui croissent dans les plaines des pays tropicaux de ces grandes parties di globe. Ce sont en général des éspèces d'hydrocotyle, l'hydrocotyle asiatica, adoensis, petiolaris, Torilis africana, Tetrapleura insularis des Iles du Cap Vert, etc. et parmis elles le daucus carota qui est certainement introduite par la culture; et pour les crucifèrs quelques éspèces de Sinapidendrum, la Koenigia intermedia, etc. On trouve des crucifères et des ombelliferes dans les Alpes de l'Abyssinie, dans le Bengal, etc., dont quelques unes descendent quelque fois dans les vallées, mais elles ne sont pas non plus en grand nombre. Il parait que les parties basses des pays tropicaux manquent presque tout'à fait de plantes de ces deux familles que je crois remplacées dans ces pays là par les capparides et par les vraies rubiacées qui s'y montrent en grand nombre et qui manquent presque tout à fait dans nos climats. J'entends ici pour vraies rubiacées les tribus de cette famille qui présentent des feuilles opposées et stipulées, telles que les ixora, les pavetta, les Cinchona etc; nous avons dans nos pays presque la seule tribus des stellatae dont $\mathrm{Mr}$ Lindley a fait une famille à part, $c^{\text {' }}$ est à dir les galium, les rubia etc qui ont des feuilles verticillées ${ }^{46}$.

Le nombre de plantes qui croissent dans l'Amerique boreale et dans l'Europe boreale vient confirmer d'une manière plus éclatante ce que Kunth à noté pour les plantes Européennes qui sont décrites dans la Flore de Pursch ${ }^{47}$. Pour ce que je sais de la végetation du nord de l'Europe j'ai pu connaitre dans la Flora boreali-americana ${ }^{48}$ de Sir W. Hooker plus de 500 espèces de plantes qui croissent dans la partie septentrionale et boréale de l'Europe. De ces 500 éspèces 320 sont des dicotyledonées, 160 des monocotyledonées, dont 113 entre cyperacées (69) et graminées (44) et 30 des cryptogames vasculaires. Il parait que la végétation de l'Amerique et de l'Europe arctiques et boreales se ressemble beaucoup, comme il est aussi de l'Asie arctique ${ }^{49}$, et que des pays tout près du pôle arctique partent pour ainsi dire tant de rayons de végétation qui uniformes dans leur naissance diffèrent toujours davantage à mesure qu'ils s'en eloignent pou differer plus tard entre eux d'une manière complète.

A propos de Saxifraga Boussingaultii, comme la plante qui de toutes le phanérogames connues s'elève le plus sur les hautes montagnes ${ }^{50}$ il est inutile de vous rappeler ici que Mr Joseph Hooker a trouvé d'autre phanérogames à une hauteur plus considerable dans le Thibet car il vous l'a ecrit dans un lettre que j'ai vu imprimée dans Hooker's Journal of botany January 1851, p. 23. Je puis ajouter que j'ai vu dans le musée botanique du Jardin de 


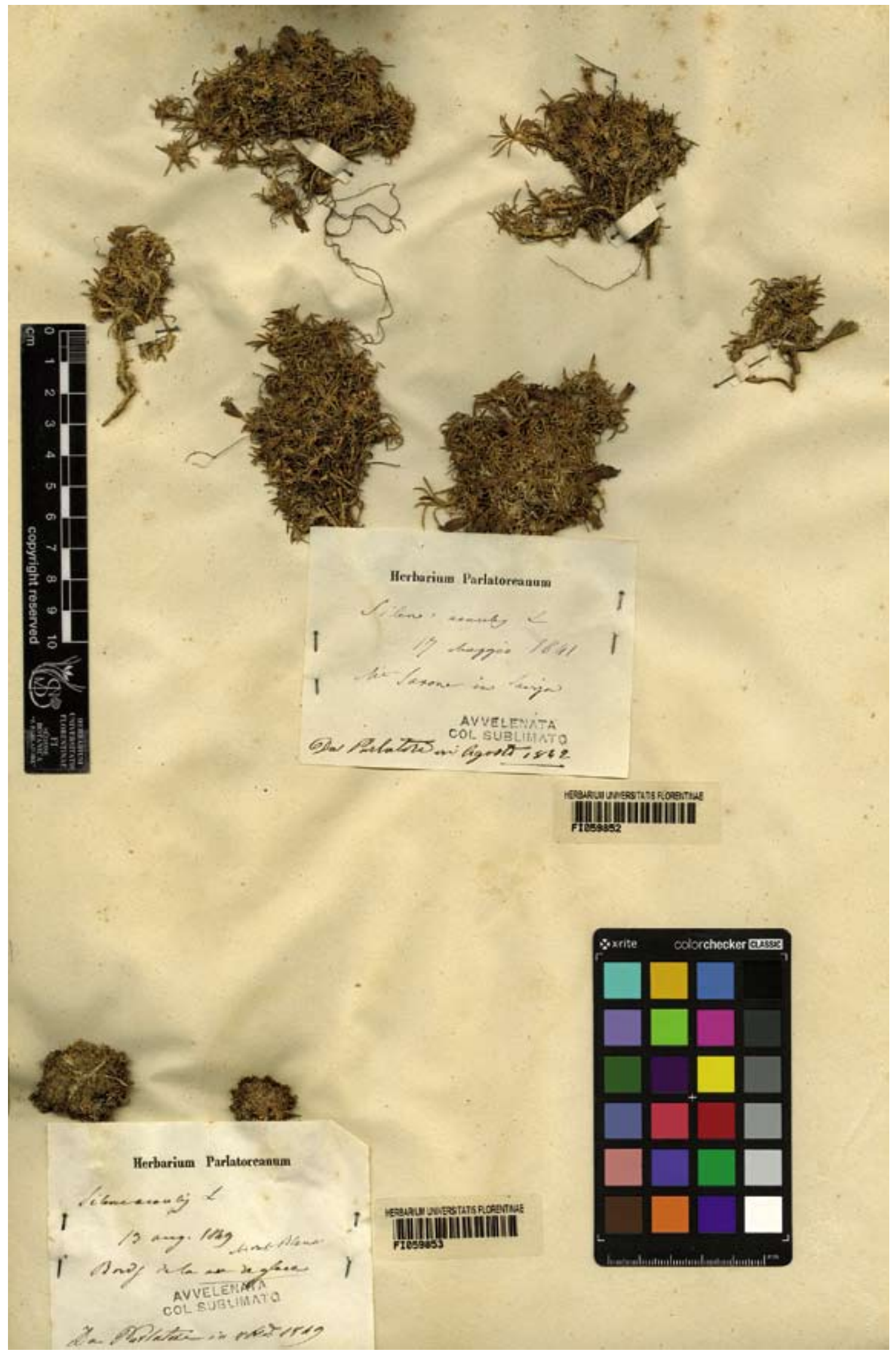

Fig. 9 - Silene acaulis L. Circum-arctic-alpine herbaceous species typical of the high altitude pioneer clods, collected by Parlatore on the edge of the Mer de Glace in the Mount Blanc Alpine range in 1849 (courtesy of the Department of Botany in the Natural History Museum of the University of Florence). / Silene acaulis L. Specie erbacea circum-artico-alpina propria delle zolle pioniere d'alta quota, raccolta da Parlatore ai margini della Mer de Glace nella catena alpina del Monte Bianco nel 1849 (per gentile concessione del Dipartimento di Botanica del Museo di Storia Naturale dell’Università di Firenze). 
Kew le rhododendrum nivale qui est l'arbuste qui s'elève le plus sur le Alpes du globe ${ }^{51}$ et qui a été trouvé par Mr. J. Hooker à Kinchin Jhou ${ }^{52}$ à 17.500 pieds, une espèce de saxifraga indeterminée de la même localité à la hauteur 16-17.000 pieds de hauteur et une alsinée également indeterminée, du Thibet qui etait en petites touffes comme nos Cherleria, Silene acaulis [Fig. 9], Aretia qui s'élèvent le plus parmi les phanerogames de l'Europe. Il parait que c'est un caractère de ces plantes de se disposer en petites touffes qui ne s'élèvent presque pas au dessus de la surface du sol; c'est ce que j'ai pu observer aussi pour les dernières plantes des Alpes de Laponie ou du voisinage di Cap Nord.

Aux arbres qui atteignent un diamètre de 20 pieds et un durée de plusieurs siècles on peut ajouter le chataigner ${ }^{53}$. Vous devez vous rappeler du fameux chataigner de l'Etna, il castagno dei cento cavalli que $\mathrm{j}$ 'ai vu dernièrement en 1845. Il a, comme on sait, le tronc divisé en cinq de sorte que on croirait d'abord que ce sont cinq arbres, mais j'ai pu m'assurer, pour quatre d'entre eux, qu'ils proviennent du même tronc. Quoique agé de plusieurs siècles, le chataigner de l'Etna continue à fleurir et à fructifier comme le fameux draconnier de l'Orotava et je conserve dans l'herbier de Florence quelques rameaux de cet arbre avec des fruits bien murs $^{54}$.

Florence ce 23 Juin 1852

Philippe Parlatore

For the letter:

Staatsbibliothek zu Berlin - Preußischer Kulturbesitz, Nachl. Alexander von Humboldt, gr. Kasten 12, Nr. 90.91, B1. 1-2

$<$ https://digital.staatsbibliothek-berlin.de/werkansicht ?PPN=PPN829064699\&PHYSID=PHYS_0001>

For the enclosure:

Staatsbibliothek zu Berlin - Preußischer Kulturbesitz, Nachl. Alexander von Humboldt, gr. Kasten 12, Nr. 90.91, B1. 3-8

$<$ https://digital.staatsbibliothek-berlin.de/werkansicht ?PPN=PPN829063579\&PHYSID=PHYS_0001>

\section{Acknowledgements}

We wish to thank Chiara Nepi, curator of the Department of Botany in the Museum of Natural History of the University of Florence, for having authorized the reproduction and publication of the illustrations of the plants and for having superintended the work carried out by the herbarium technicians, Anna Donatelli and Lorenzo Lastrucci, in the Herbarium Centrale Italicum in identifying the plants referred to by Parlatore in the correspondence; the Stiftung Stadtmuseum Berlin for authorizing the publication of Humboldt's portrait; Eliana Calandra, director of Palermo City Library for kindly sending, through Alexander Di Bartolo, the scanned versions of the letters of Humboldt to Parlatore; Elvira Cantarella of the University of Milan for her precious advice and attentive reading of the manuscript; Filippo Bianconi, geologist and historian of geology, for his help in deciphering the part in German in Humboldt's notes.

\section{NOTES}

${ }^{1}$ It is not always easy to understand the meaning attributed to the climate by scientists from time to time. Somewhat schematically, it is possible to say that it used to be defined as the result of measuring the meteorological conditions (temperature, humidity, atmospheric pressure) of a region or place in one given moment in time, and not, as is the practice now, over a longer period of time. On the other hand, the climate was also considered as it is now, as zones characterized by particular, diverse environmental features (a hot, temperate, cold climate, etc.).

${ }^{2}$ As is inferred from a letter to Humboldt of 20 July 1844 from Leopold II Grand Duke of Tuscany, which reads as follows: "Très cher Comte, Le professeur de botanique Philippe Parlatore se rend à Berlin. Il m'est impossible de le laisser partir sans le charger d'une lettre pour vous, cher comte, qui exprime mes remerciments pour les recommandations que vous m'avez faites pour que la Toscane pût s'enrichir de plusieurs hommes illustres. Vous, le père et le protecteur de toutes les sciences naturelles, connaissiez M. Parlatore, et un jugement porté par vous suffisait; il est à Florence [...]" (Bianchi, 1874: 73-74); see also Parlatore, 1992: 103.

${ }^{3}$ Unlike the journey to the Mount Blanc mountain range, which was carried out at Parlatore's own expense, this journey was funded by Grand Duke Leopold II for a considerable sum (Parlatore, 1992: 188-189).

${ }^{4}$ The botanical part - which was to be dealt with in the second part of volume five of Kosmos of which the first four volumes were published between 1845 and 1858 and the first part of volume five posthumously in 1862 - was never published (Parlatore, 1860a: 36; Beck, 1961: 228; Bowen, 2009: 247).

${ }^{5}$ A reference to Candolle A. P. de, 1840 and to Candolle A. de, 1855 , a work still in progress at the time, the basic outline of which Humboldt must certainly have been aware. It is believed that the heart of the matter could have been the German scientist's fear of being obscured by the new direction given to botanical geography by the two Candolles, in particular by Alphonse.

${ }^{6}$ Humboldt is referring to Forbes' work on Fauna and Flora of British isles (Forbes, 1846), and to the pre-eminent role that the English naturalist assigned to geology for an understanding of the actual distribution of vegetation. For Humbold's contradictory convictions concerning the usefulness of geology the reference is to Jackson, 2009: 20-21.

${ }^{7}$ The literature is particularly extensive on this topic, in connection to Humboldt's organicistic concept of nature: Cambiano, 1997; Segala, 1998; Bowen, 2009: 222-233; Frigo, 2020 and the bibliography cited therein, to mention only a few. There is also a mention in Parlatore, 1860a: 36-37, in particular in relation to the first two volumes of $\mathrm{Ko}$ smos.

${ }^{8}$ Humboldt's words quoted by Parlatore sound more like encouragement rather than opinion since the Sicilian botanist had not yet published anything in the field of phytogeography, except a part relative to the altitudinal zones mentioned previously in Parlatore, 1850 . He would deal with this subject only later. In this regard, see Parlatore, 1857 ; 1858a, 1859, 1860b, 1878. Moreover, in the Biblioteca Comunale di Palermo (Palermo City Library), where, as a bequest, the manuscripts of the Sicilian naturalist are preserved, there are two voluminous bundles of manuscripts entitled Geografia botanica dell'Italia (Fondo Parlatore, 5 Qq F 30-31) and another just as voluminous entitled Geografia botanica universale (Fondo Parlatore, 5 Qq F 33).

${ }^{9}$ The letter is published in Rodolico, 1968. For this letter and Humboldt's two successive letters to Parlatore, which are reproduced here, the author had purposely omitted any note of criticism or scientific 
comment, stating that he wished to leave "attentive scientific analysis to a historian of botany" (Rodolico, 1968: 115).

${ }^{10}$ He left Berlin on 24 May (Parlatore, 1992: 191).

${ }^{11}$ The reference is to Flora italiana, ossia descrizione delle piante che crescono spontanee o vegetano come tali in Italia e nelle isole ad essa aggiacenti disposta secondo il metodo naturale (Firenze, Le Monnier, Tip. dei Succ. Le Monnier, Stab. tip. fiorentino), a monumental compendium of the flora of Italy which Parlatore was nevertheless unable to finish. The first volume was published in 1848 and successive volumes were published by Parlatore between 1852 and 1875. After his death (1877), his work was continued by his pupil Teodoro Caruel up to the tenth and final volume (1894).

${ }^{12}$ The literature is extensive concerning Humboldt's aspirations to ascribe natural phenomena to universal laws and includes, among others: Milanesi,1975; Farinelli, 1981 and the bibliography cited therein, Vallino, 1986; Greppi, 1992; Bowen, 2009: 220, 221 and 223; Frigo, 2020 and the bibliography cited therein.

${ }^{13}$ The date of dispatch of this lithograph is not certain. It is preserved in the Sezione Botanica del Museo di Storia Naturale dell'Università di Firenze (Department of Botany in the Museum of Natural History of the University of Florence). For the location of other specimens of this lithograph see Nelken, 1980: 138.

${ }^{14}$ Parlatore uses the term vegetation, which at the time was not distinct from that of flora.

Vegetation and flora are now two separate concepts: the former describes how plant communities or plant associations appear and how they are organized in relation to the climate, the substratum and the external forces determining changes and transformations; the latter is concerned with the number and quality of the plant species present in a region in relation to their biology, ecology and geographic distribution, independent of communities or associations. To be functional to the explanation that the scientist from Palermo wants to offer Humboldt, the rays of vegetation evoked by Parlatore must be interpreted as rays of flora, otherwise any possibility of comparison within the framework of Humboldt's enquiries would fail.

${ }^{15}$ See note 9 .

${ }^{16}$ The water features of Potsdam were well-known thanks to the works carried out by Frederick William IV of Prussia, who introduced a steam engine to make them function.

${ }^{17}$ Guillemin et al., 1830-1833.

${ }^{18}$ Brown, 1818.

${ }^{19}$ Parlatore's answer is the rather long and detailed list (enclosure to Letter 2) of the Poaceae (grasses) and Cyperaceae (sedges) which grow both in the Italian peninsula and at the Tropics. The list aims to demonstrate to Humboldt that, without the dicotyledons, these two families alone are not enough to differentiate between temperate zones and tropical zones. Therefore, they are not a discriminant in distinguishing temperate from tropical climates.

${ }^{20}$ A reference to Frederick William IV of Prussia.

${ }^{21}$ See Letter 4 for the conferment of this honour.

${ }^{22}$ See note 8.

${ }^{23}$ A reference to Tenore, 1827: 80.

${ }^{24}$ On the North-American origins of Erigeron canadensis and its probably accidental introduction by humans into Europe, see, among others, Linnaei, 1764: 1210; Richard, 1828: 335.; Bourguet, 1992: 348.

${ }^{25}$ For a comment on the work, see Beck, 1989: 167-255.

${ }^{26}$ A reference to Humboldt, Bonpland \& Kunth, 1815-1825.

${ }^{27}$ Alfred von Reumont (1808-1887), scholar and Prussian diplomat, was ambassador in Florence between 1851 and 1860.

${ }^{28}$ Humboldt is referring to Frederick William IV.

${ }^{29}$ A reference to Parlatore's travels to the Alpine range (Parlatore, $1850)$.
${ }^{30}$ The letter written by Frederick William IV of Prussia to Parlatore bears the date of 24 November 1851. A photographic reproduction of the letter was published by Alaimo, 1956.

${ }^{31}$ This is probably an allusion to the sentencing to death of Luigi Settembrini by Ferdinand II of Bourbon (I February 1851) and the successive commutation of the sentence into life imprisonment.

${ }^{32}$ Vincenzo Antinori, director of the Museum of Physics and Natural History in Florence from 1829 to 1859.

${ }^{33}$ This may be a reference to Leopoldo Nobili, professor of Physics at the Museum of Physics and Natural History in Florence, of whose death (1835) Humboldt may not necessarily have been aware.

${ }^{34}$ Abramo Basevi (1818-1885), Tuscan doctor and musician.

${ }^{35}$ Parlatore is referring to the Giornale Botanico Italiano founded by the Section of Botany of the Congress of Italian Scientists in Padua, under the direction of Alberto Bracht and himself (Atti della quarta Riunione degli scienziati tenuta in Padova nel settembre del 1842, 1843: 340-342). On the example of journals already published north of the Alps for many years, it was intended to group together all the publications of botanists with the aim of facilitating their research and keeping them updated in a constant and permanent manner on what was being published in Italy, thus reducing the need to meet periodically. The first issue was published in Forence in 1844 but publication was interrupted in 1852, as Parlatore wrote, "as there were not enough subscribers to cover the expenses, I had to give up what I had embarked on with a personal loss of over two thousand lira" (Parlatore, 1992: 208). The journal returned only many years later, in 1862, with the title Nuovo giornale botanico under the direction of the Florentine naturalist Odoardo Beccari.

${ }^{36}$ See note 8.

${ }^{37}$ See Parlatore F., 1858b, which completes the Italian monocotyledons dealt with in the first two volumes.

${ }^{38}$ Parlatore is referring to his intention to publish a second volume of his travels in the Scandinavian peninsula (Parlatore 2012: back cover) which, however, was never published.

${ }^{39}$ See Letter 4. The reference here is to the letter from Frederick William IV of Prussia to Parlatore.

${ }^{40}$ Brown, 1818.

${ }^{41}$ Humboldt, 1836: 76.

${ }^{42}$ Buffon, 1830: 359-418.

${ }^{43}$ Parlatore's answer clearly shows how no law exists to regulate the floristic composition of a region and emphasizes how composition should be interpreted as the sum of two contingents of species: A) those differentiating in the region itself or arriving from outside in the initial expansionary phase of their populations in a pre-human era (autochthonous species); B) species introduced directly or indirectly into the territory by humans and defined as non-native or alien species.

${ }^{44}$ Parlatore explains how the definition of cosmopolitan cannot correspond to a concept of absolute value. It is now known that certain cryptogams (mosses, liverworts and ferns) can be considered cosmopolitan but only if reference is made to restricted and isolated regions of political but not physical geography because their habitats do not cover the land surface in a continuous way and, in fact, vast uninhabitable areas, intercalated with inhabitable areas, exist for each species. Moreover, as Parlatore states, no plant species with seeds grows in the Antarctic and at the same time in Central America, in China or in the Mediterranean area.

${ }^{45}$ See note 38 .

${ }^{46}$ Before the research carried out by John Lindley, the Rubiaceae, to which Galium belongs, were included in an aggregate of plants having flowers with gamopetalous and actinomorphic corollas, ascribed to the "alliance Stellales" and "order Stellatae" (Lindley, 1836: 249). In sharing this view, Parlatore wanted to draw attention to Lindley's scientific correctness in having identified, within the Stellatae, the more 
homogeneous and - in modern terms - phylogenetically coherent subgroup, which corresponds to the Rubiaceae family.

${ }^{47}$ Pursh, 1814.

${ }^{48}$ Hooker, 1829.

${ }^{49}$ Hooker, 1829: IV; Kunth, 1847: 530; Parlatore, 1857. The similarity between two or more floras can now be assessed quantitatively and therefore more objectively by means of mathematical indices, premature for the times but consistent with the considerations made by Pursh and Kunth, which Parlatore shared.

${ }^{50}$ Humboldt, 1851: 162.

${ }^{51}$ Hooker, 1849: tab. XXVI.

${ }^{52}$ Today known as Kanchenjunga, a mountain situated between Nepal and Sikkim in India, territories explored by Hooker between 1848 and 1849.

${ }^{53}$ Parlatore refers to Humboldt (1851: 443-457), where the Castagno dei cento cavalli (Hundred Horse Chestnut) (Etna, Sicily) is not listed among the trees famous for their size and rarity.

${ }^{54}$ Parlatore, 1992: 132.

\section{REFERENCES}

Alaimo M. E., 1956 - Tra le carte inesplorate di un trionfatore di congressi. In: Diario della XLVI riunione della Società Italiana per il Progresso delle Scienze, s.i.t: 12-13.

Atti della quarta Riunione degli scienziati tenuta in Padova nel settembre del 1842, 1843, Co'tipi del Seminario, Padova.

Bartolucci F., Peruzzi L., Galasso G., Albano A., Alessandrini A., Ardenghi N. M. G., Astuti G., Bacchetta G., Ballelli S., Banfi E., Barberis G., Bernardo L., Bouvet D., Bovio M., Cecchi L., Di Pietro R., Domina G., Fascetti S., Fenu G., Festi F., Foggi B., Gallo L., Gottschlich G., Gubellini L., Iamonico D., Iberite M., JiménezMejías P., Lattanzi E., Marchetti D., Martinetto E., Masin R. R., Medagli P., Passalacqua N. G., Peccenini S., Pennesi R., Pierini B., Poldini L., Prosser F., Raimondo F. M., Roma-Marzio F., Rosati L., Santangelo A., Scoppola A., Scortegagna A., Selvaggi A., Selvi F., Soldano A., Stinca A., Wagensommer R. P., Wilhalm T. \& Conti F., 2018 - An updated checklist of the vascular flora native to Italy. Plant Biosystems 152 (2): 179-303. < https://doi.org/10.1080/1126 3504.2017.1419996>

Beck H., 1961 - Alexander von Humboldt. Steiner Verlag GMBH, Wiesbaden.

Beck H. (herausgegeben und kommentiert von), 1989 Alexander von Humboldt Schriften zur Geographie der Pflanzen. Wissenschaftliche Buchgesellschaft, Darmstadt.

Béguinot A., 1906 - Pensieri intorno alla storia dello sviluppo e allo stato attuale della geografia botanica. Bollettino della Società Geografica Italiana, ser. 4, 7 (11): 1052-1065.

Bianchi N., 1874 - Carlo Matteucci e l'Italia del suo tempo. V. Bona, Torino.

Bourguet M.-N., 1992 - L'esploratore. In: L'uomo del Settecento. Vovelle M. (a cura di). Laterza \& figli, Roma-Bari: 283-351.

Bowen M., 2009 - Empiricism and geographical thought from Francis Bacon to Alexander von Humboldt. Cambridge University Press, Cambridge.

Brown R., 1818 - Observations, systematical and geographical, on Professor Christian Smith's Collection of Plants from the Vicinity of the River Congo. In: Narrative of an Expedition to explore the River Zaire usually called the Congo in South Africa, in 1816. Tuckey J. H., \& Smith Ch. (under the direction of). Murray, London, Appendix n .V: 420-485.

Buffon G.-L. Leclerc de, 1830 - Oeuvres complètes. Pillot, Paris, vol. XV.

Cambiano G., 1997 - Alexander von Humboldt e il sapere degli antichi. In: Politica, scienze e cosmopolitismo Alexander e Wihelm von Humboldt. Malandrino C. (a cura di). Franco Angeli, Milano: 194-208.

Candolle A. de, 1855 - Géographie botanique raisonnée. Masson, Paris Kessmann, Genève.

Candolle A. P. de, 1840 - Essai elementaire de géographie botanique. Levrault, Strasbourg.
Candolle A. P. de, 1844 - Théorie élémentaire de la botanique (par Candolle A. de). Roret, Paris.

Farinelli F., 1981 - Storia del concetto geografico di paesaggio. In: Paesaggio immagine e realtà. Electa, Milano: 151-158.

Forbes E., 1846 - On the connexion between the distribution of the existing Fauna and Flora of the British Isles, and the geological changes which have affected their area, especially during the epoch of the northern drift. Longman, Brown, Green and Longmans, London.

Frigo G. F., 2020 - Empirics, science, aesthetics: Alexander von Humboldt's original approach to Nature, Bulletin of Environmental Life Sciences, 2: 79-90. < doi: 10.15167/2612-2960/ BELS2020.2.1.1231>

Greppi C. (a cura di), 1992 -Alexander von Humboldt L'invenzione del Nuovo mondo. La Nuova Italia, Scandicci (Firenze): XXXI-LIV.

Grisebach A, 1872 - Die Vegetation der Erde nach ihrer klimatischen Anordnung. Engelmann, Leipzig, vol. I.

Guillemin J.-A., Perrottet S. \& Richard A., 1830-1833 - Florae Senegambiae tentamen, seu Historia plantarum in diversis Senegambiae regionibus a peregrinatoribus Perrottet et Leprieur detectarum. Treuttel et Wurtz, Parisiis, vol. I.

Halácsy E. v., 1902 - Theodor von Heldreich Ein Nachruf. Magyar Botanikai Lapok, I: 325-336.

Haynald L., 1879 - Denkrede auf Philipp Parlatore. Franklich Verein, Budapest.

Heldreich T. di, 1843 - Descrizione di alcune specie nuove di piante siciliane. Annali dell'Accademia degli aspiranti naturalisti di $\mathrm{Na}$ poli, I: 283-292.

Hooker J. D., 1849 - The Rhododendrons of Sikkim-Himalaya: being an account botanical and geographical of the rhododendrons recently discovered in the mountains of eastern Himalaya, from drawings and descriptions made on the spot during a government botanical mission to that country. Reeve, Bentham and Reeve, London, n. 29, tab XXVI.

Hooker J. D., 1851 - Letter to Baron Humboldt, Kossya Mountains, Sept. $23^{\text {rd }} 1850$. Hooker's Journal of botany and Kew Garden miscellany, v. 3: 23-31.

Hooker W. J., 1829 - Flora boreali-americana. H. G. Bohn, London, vol. I.

Humboldt A. de, 1805 - Essai sur la géographie des plantes. Schoell, Paris.

Humboldt A. de (redigé par), 1814 - Voyage aux Régions Équinoxiales du Nouveau Continent fait en 1799, 1800, 1801, 1802, 1803 et 1804 par A de Humboldt et A. Bonpland. Schoell, Paris, vol. I.

Humboldt A. von, 1817 - De distributione geographica Plantarum secundum coeli temperiem et altitudinem montium. Prolegomena. In Libraria-graeco-latino-germanica, Lut. Paris.

Humboldt A. de, 1837 - Examen critique de l'Histoire et de la Géographie du Nouveau Continent. Gide, Paris, tome II.

Humboldt A. de, 1851 - Tableaux de la nature. Gide et Baudry, Paris, tome II.

Humboldt A. von, [1851] - Alexander von Humboldt an Christian Gottfried Ehrenberg. [Berlin], [13. Mai 1851], hg. v. Anette Wendt unter Mitarbeit von Eberhard Knobloch. In: edition humboldt digital, hg. v. Ottmar Ette. Berlin-Brandenburgische Akademie der Wissenschaften, Berlin. Version 5 vom 11.09.2019. <https://edition-humboldt.de/v5/H0016601>

Humboldt A. von, Bonpland A. \& Kunth C. S., 1815-1825 - Nova genera et species plantarum quas in peregrinatione ad plagam aequinoctialem orbis novi collegerunt. Apud Gide filium, Maze, Sumtibus Librairie Graeco-Latino-Germanicae, Lutetiae Parisiorum.

IPNI, 2020 - International Plant Names Index. Published on the Internet $<$ http:/www.ipni.org $>$

Jackson S. T, 2009 - Introduction. In: Essay on the Geography of Plants (edited by Jackson S. T.). Alexander von Humboldt and Aimé Bonpland. University of Chicago Press, Chicago and London.

Kunth K. S., 1847 - Lehrbuch der Botanik, Dunkler und Humblot, Berlin, Erster Teil.

Lindley J., 1836 - Natural System of Botany. Longman, Rees, Orme, Brown, Green and Longman, London.

Linnaei C., 1764 - Species plantarum, exhibentes plantas rite cognitas, ad genera relatas [...] digestas. Trattner, Vindobonae, tom. II. 
Milanesi M., 1975 - Introduzione. In: Alexander von Humboldt, La geografia, i viaggi Antologia degli scritti. Milanesi M. \& Visconti Viansson A. (a cura di). Franco Angeli, Milano: 7-29.

Moggi G., 1978 - Filippo Parlatore nel centenario della morte. Il Naturalista Siciliano, s. 4, 2: 97-108.

Moggi G., 2009 - Storia delle collezioni botaniche del Museo. In: Il Museo di Storia Naturale dell'Università di Firenze. Raffaelli M. (a cura di). Firenze University Press, Firenze, vol. II: 3-57.

Negri G., 1927 - Commemorazione di Filippo Parlatore nel cinquantenario della sua morte. Nuovo Giornale Botanico Italiano, n.s., 34: 972-999.

Nelken H., 1980 - Alexander von Humboldt Bildnisse und Künstler Eine Dokumentierte Ikonographie, Reimer, Berlin.

Parlatore F., 1846 - Sopra un nuovo scopo a cui potrebbe destinarsi una parte de' Giardini Botanici. Giornale botanico italiano, a. II, p. I, t. 1: 167-175.

Parlatore F., 1850 - Viaggio alla catena del Monte Bianco e al Gran San Bernardo. Le Monnier, Firenze.

Parlatore F., 1857 - Studi sulla geografia botanica. Annuario dell'I. R. Museo di Fisica e Storia Naturale: 163-185.

Parlatore F., 1858a - Studi sulla geografia botanica. Annuario dell'I. $R$. Museo di Fisica e Storia Naturale: 199-205.

Parlatore F., 1858b - Flora italiana, ossia descrizione delle piante che crescono spontanee o vegetano come tali in Italia e nelle isole ad essa aggiacenti disposta secondo il metodo naturale. Le Monnier, Firenze, vol. III.

Parlatore F., 1859 - Studi sulla geografia botanica. Annuario dell'I. $R$. Museo di Fisica e Storia Naturale: 199-208.

Parlatore F., 1860a - Elogio di Alessandro Humboldt. Le Monnier, Firenze.

Parlatore F., 1860b - Studi sulla geografia botanica. Annuario dell'I. R. Museo di Fisica e Storia Naturale: 123-132.

Parlatore F., 1878 - Études sur la géographie botanique de l'Italie. Baillière, Paris.

Parlatore F., 1992 - Mie memorie. (a cura di A. Visconti). Sellerio, Palermo.

Parlatore F., 2012 - Viaggio per le parti settentrionali di Europa fatto nell'anno 1851. (a cura di Di Bartolo A. con introduzione di Visconti A.). Ibis, Como-Pavia.

Pignatti S., Guarino R., La Rosa M., 2017-2019 - Flora d'Italia. Edagricole, Bologna.

Portal of the Flora of Italy, 2020 - Available at $<$ http:/dryades.units.it/ floritaly>

Pursh F. T., 1814 - Flora Americae Septentrionalis, or, A systematic arrangement and description of the plants of North America: containing, besides what has been described by preceding authors, many new and rare species collected during twelve years' travels and residence in that country. White, Cochrane, London.

Reveal J. L., 2011 - Indices Nominum Supragenericorum Plantarum Vascularium. University of Maryland, The New York Botanical Garden. $<$ http://www.plantsystematics.org/reveal/pbio/fam/allspgnames.html>

Richard A., 1828 - Nouveaux Élements de Botanique. Bechet Jeune, Paris.

Rodolico F, 1968 - Per la storia della fitogeografia: lettere di Alessandro von Humboldt a Filippo Parlatore. Physis Rivista internazionale di storia della scienza, anno X, fasc. 2: 113-118.

Rodolico F., 1997 - Naturalisti esploratori dell'Ottocento italiano. Le Monnier, Firenze.

Segala M., 1998 - Riflessioni sulla scienza romantica. Nuncius Annali di Storia della Scienza, XIII, 1998, fasc. 2: 677-691.

Stevens H., [1863] - Catalogue of the Humboldt Library. [Stevens, London].

Stevens P. F., 2020 - Angiosperm Phylogeny Website. <http://www. mobot.org/MOBOT/research/APweb>

Tenore M., 1827 - Cenno sulla geografia fisica e botanica del Regno di Napoli. Zambaja, Napoli.

Tirrito L., 1882 - Sulla vita e sulle opere di Filippo Parlatore. Virzì, Palermo.

Tomaselli R., 1956 - Introduzione allo studio della fitosociologia. Poligrafica lombarda, Milano.
Turland N. J., Wiersema, J. H., Barrie, F. R., Greuter, W., Hawksworth, D. L., Herendeen, P. S., Knapp, S., Kusber, W.-H., Li, D.-Z., Marhold, K., May, T. W., McNeill, J., Monro, A. M., Prado, J., Price, M. J. \& Smith, G. F. (eds.), 2018 - International Code of Nomenclature for algae, fungi, and plants (Shenzhen Code) adopted by the Nineteenth International Botanical Congress Shenzhen, China, July 2017. Regnum Vegetabile 159. Koeltz Botanical Books, Glashütten. <https://doi.org/10.12705/Code.2018>

Vallino F. O., 1986 - Presentazione. In: Alexander von Humboldt Viaggio alle regioni equinoziali del nuovo continente fatto negli anni 11799, 1800, 1801, 1802, 1803 e 1804. Vallino F. O. (a cura di). Palombi, Roma, tomo I: IX-XCIII.

Visconti A., 1987 - Per la storia della botanica in Italia: le Memorie inedite di Filippo Parlatore. In: Atti del Convegno "I naturalisti e la cultura scientifica siciliana nell'800". Stampatori tipolitografi associati, Palermo: 197-206.

Visconti A, 2004 - The Journeys of the Sicilian Botanist Filippo Parlatore (1816-1877). Proceedings of the California Academy of Sciences. 55, suppl. II: 29-41. 
TAXONOMIC TABLE OF PLANTS MENTIONED IN THE CORRESPONDENCE

\begin{tabular}{|c|c|c|c|}
\hline Original indication & Family & Current name & Reference \\
\hline Abrus precatorius & Fabaceae & Abrus precatorius L. & P5A \\
\hline Achillea millefolium & Asteraceae & Achillea millefolium $\mathrm{L}$. & P5A \\
\hline Agropyron junceum & Poaceae & $\begin{array}{l}\text { Thinopyrum junceum (L.) Á.Löve (= Elymus farctus } \\
\text { (Viv.) Runemark ex Melderis) }\end{array}$ & $\mathrm{P} 2 \mathrm{~A}$ \\
\hline Aira caryophyllea & Poaceae & Aira caryophyllea $\mathrm{L}$. & P5A \\
\hline Aira caryophyllea & Poaceae & Aira caryophyllea $\mathrm{L}$. & $\mathrm{P} 2 \mathrm{~A}$ \\
\hline Alopecurus geniculatus & Poaceae & Alopecurus geniculatus L. & $\mathrm{P} 2 \mathrm{~A}$ \\
\hline Alopecurus pratensis & Poaceae & Alopecurus pratensis $\mathrm{L}$. & $\mathrm{P} 2 \mathrm{~A}$ \\
\hline Alsine media & Caryophyllaceae & Stellaria media L. & HP2B \\
\hline Alsinée & Caryophyllaceae & Alsineae Lam. \& DC. & P5A \\
\hline Ammophila arundinacea & Poaceae & $\begin{array}{l}\text { Calamagrostis arenaria (L.) Roth subsp. } \\
\text { arundinacea (Husn.) Banfi, Galasso \& Bartolucci }\end{array}$ & $\mathrm{P} 2 \mathrm{~A}$ \\
\hline Andropogon hirtum & Poaceae & Hyparrhenia hirta (L.) Stapf subsp. hirta & $\mathrm{P} 2 \mathrm{~A}$ \\
\hline Andropogon ischaemum & Poaceae & Bothriochloa ischaemum (L.) Keng & $\mathrm{P} 2 \mathrm{~A}$ \\
\hline Anthoxanthum odoratum & Poaceae & Anthoxanthum odoratum L. & P2A, P5A \\
\hline Aretia & Primulaceae & Androsace sp. & P5A \\
\hline Aretia helvetica & Primulaceae & Androsace helvetica (L.) All. & HP2 \\
\hline Aretia imbricata & Primulaceae & Androsace helvetica (L.) All. & HP2 \\
\hline Argemone mexicana & Papaveraceae & Argemone mexicana $\mathrm{L}$. & P5A \\
\hline Avenella flexuosa & Poaceae & Avenella flexuosa (L.) Drejer subsp. flexuosa & P2A, P5A \\
\hline Avicennia tomentosa & Acanthaceae & Avicennia germinans (L.) L. & $\mathrm{P} 5 \mathrm{~A}$ \\
\hline Beckmannia erucaeformis & Poaceae & Beckmannia eruciformis (L.) Host & $\mathrm{P} 2 \mathrm{~A}$ \\
\hline Briza maxima & Poaceae & Macrobriza maxima (L.) Tzvelev & $\mathrm{P} 2 \mathrm{~A}$ \\
\hline Briza minor & Poaceae & Briza minor $\mathrm{L}$. & $\mathrm{P} 2 \mathrm{~A}$ \\
\hline Cactus? Enna & Cactaceae? & Opuntia sp.? & HP2B \\
\hline Callitriche verna & Callitrichaceae & Callitriche palustris L. & P5A \\
\hline Canna indica & Cannaceae & Canna indica $\mathrm{L}$. & HP2 \\
\hline Capsella bursa pastoris & Brassicaceae & Capsella bursa-pastoris L. & P5A \\
\hline Cardamine hirsuta & Brassicaceae & Cardamine hirsuta L. & P5A \\
\hline $\begin{array}{l}\text { Cardiospermum } \\
\text { halicacabum }\end{array}$ & Sapindaceae & Cardiospermum halicacabum L. & P5A \\
\hline Carex curta & Cyperaceae & Carex canescens $\mathrm{L}$. & P5A \\
\hline Carex festiva & Cyperaceae & Carex macloviana d'Urv. & P5A \\
\hline Cassia occidentalis & Fabaceae & Senna occidentalis (L.) Link & P5A \\
\hline Catabrosa aquatica & Poaceae & Catabrosa aquatica (L.) P.Beauv. & $\mathrm{P} 2 \mathrm{~A}$ \\
\hline Cerastium arvense & Caryophyllaceae & Cerastium arvense $\mathrm{L}$. & P5A \\
\hline Cerastium vulgatum & Caryophyllaceae & Cerastium glomeratum Thuill. & P5A \\
\hline Chaetaria adscensionis & Poaceae & Aristida adscensionis L. & $\mathrm{P} 2 \mathrm{~A}$ \\
\hline
\end{tabular}




\begin{tabular}{|c|c|c|c|}
\hline Original indication & Family & Current name & Reference \\
\hline Cherleria & Caryophyllaceae & $\begin{array}{l}\text { Cherleria sp. + Facchinia sp. + Minuartia sp. }+ \\
\text { Sabulina } \text { sp. }\end{array}$ & P5A \\
\hline Chrysobalanus icaco & Chrysobalanaceae & Chrysobalanus icaco L. & P5A \\
\hline Cinchona rubiacee & Rubiaceae & Cinchona sp. & P5A \\
\hline Cladium mariscus & Cyperaceae & Cladium mariscus (L.) Pohl & $\mathrm{P} 2 \mathrm{~A}$ \\
\hline Conocarpus erecta & Combretaceae & Conocarpus erectus L. & P5A \\
\hline Crocifere & Brassicaceae & Brassicaceae Burnett & P5A \\
\hline Cynodon dactylon & Poaceae & Cynodon dactylon (L.) Pers. & $\mathrm{P} 2 \mathrm{~A}$ \\
\hline Cynosurus echinatus & Poaceae & Cynosurus echinatus L. & $\mathrm{P} 2 \mathrm{~A}$ \\
\hline Cyperus difformis & Cyperaceae & Cyperus difformis $\mathrm{L}$. & $\mathrm{P} 2 \mathrm{~A}$ \\
\hline Cyperus globosus & Cyperaceae & Cyperus flavidus Retz. & $\mathrm{P} 2 \mathrm{~A}$ \\
\hline Cyperus mucronatus & Cyperaceae & Cyperus laevigatus L. & $\mathrm{P} 2 \mathrm{~A}$ \\
\hline Cyperus polystachyus & Cyperaceae & Cyperus polystachyos Rottb. & $\mathrm{HP} 2, \mathrm{P} 2 \mathrm{~A}$ \\
\hline Cyperus rotundus & Cyperaceae & Cyperus rotundus L. & $\mathrm{P} 2 \mathrm{~A}$ \\
\hline Dactylis glomerata & Poaceae & Dactylis glomerata L. & $\mathrm{P} 2 \mathrm{~A}$ \\
\hline $\begin{array}{l}\text { Dactyloctenium } \\
\text { aegyptiacum }\end{array}$ & Poaceae & Dactyloctenium aegyptium (L.) Willd. & $\mathrm{P} 2 \mathrm{~A}$ \\
\hline Danthonia provincialis & Poaceae & Danthonia alpina Vest & $\mathrm{P} 2 \mathrm{~A}$ \\
\hline Daucus carota & Apiaceae & Daucus carota $\mathrm{L}$. & P5A \\
\hline Digitaria sanguinalis & Poaceae & Digitaria sanguinalis (L.) Scop. & $\mathrm{P} 2 \mathrm{~A}$ \\
\hline Dinebra arabica & Poaceae & Dinebra retroflexa (Vahl) Panz. & $\mathrm{P} 2 \mathrm{~A}$ \\
\hline Dodonea viscosa & Sapindaceae & Dodonaea viscosa (L.) Jacq. & P5A \\
\hline Draba incana & Brassicaceae & Draba stylaris J.Gay ex W.D.J.Koch & P5A \\
\hline Ehrharta panicea Smith & Poaceae & Ehrharta erecta Lam. & $\mathrm{P} 2 \mathrm{~A}$ \\
\hline $\begin{array}{l}\text { Eleocharis atropurpurea } b . \\
\text { minor }\end{array}$ & Cyperaceae & Eleocharis atropurpurea (Retz.) J.Presl \& C.Pres1 & $\mathrm{P} 2 \mathrm{~A}$ \\
\hline Eleocharis palustris & Cyperaceae & Eleocharis palustris (L.) Roem. \& Schult. & P5A \\
\hline Epilobium tetragonum & Onagraceae & Epilobium tetragonum $\mathrm{L}$. & P5A \\
\hline Eragrostis megastachya & Poaceae & $\begin{array}{l}\text { Eragrostis cilianenesis (All.) Vignolo ex Janchen } \\
\text { subsp. cilianensis }\end{array}$ & $\mathrm{P} 2 \mathrm{~A}$ \\
\hline Erigeron alpinus & Asteraceae & Erigeron alpinus $\mathrm{L}$. & P5A \\
\hline Erigeron canadense & Asteraceae & Erigeron canadensis L. & HP3 \\
\hline Erigeron uniflorus & Asteraceae & Erigeron uniflorus L. & P5A \\
\hline $\begin{array}{l}\text { Fimbristylis annua Roem? } \\
\text { e Schult. }\end{array}$ & Cyperaceae & Fimbristylis annua (All.) Roem. \& Schult. & $\mathrm{P} 2 \mathrm{~A}$ \\
\hline Fimbristylis dichotoma & Cyperaceae & Fimbristylis dichotoma (L.) Vahl subsp. dichotoma & $\mathrm{P} 2 \mathrm{~A}$ \\
\hline Fimbristylis squarrosa Vahl & Cyperaceae & Fimbristylis squarrosa Vahl & $\mathrm{P} 2 \mathrm{~A}$ \\
\hline Funaria hygrometrica & Funariaceae & Funaria hygrometrica Hedw. & P5A \\
\hline Galium & Rubiaceae & Galium sp. & P5A \\
\hline Galium aparine & Rubiaceae & Galium aparine L. & P5A \\
\hline Gentiana tenella & Gentianaceae & Gentianella tenella (Rottb.) Börner & P5A \\
\hline
\end{tabular}




\begin{tabular}{|c|c|c|c|}
\hline Original indication & Family & Current name & Reference \\
\hline Glyceria aquatica & Poaceae & Catabrosa aquatica (L.) P.Beauv. & $\mathrm{P} 2 \mathrm{~A}$ \\
\hline Guilandina bonduc & Fabaceae & Guilandina bonduc L. & $\mathrm{P} 5 \mathrm{~A}$ \\
\hline Holcus lanatus & Poaceae & Holcus lanatus L. & $\mathrm{P} 2 \mathrm{~A}$ \\
\hline Holcus mollis & Poaceae & Holcus mollis $\mathrm{L}$. & $\mathrm{P} 2 \mathrm{~A}$ \\
\hline Hordeum maritimum & Poaceae & Hordeum marinum Huds. & $\mathrm{P} 2 \mathrm{~A}$ \\
\hline Hordeum murinum & Poaceae & $\begin{array}{l}\text { Hordeum murinum L. (+ subsp. leporinum (Link) } \\
\text { Arcang.) + subsp. glaucum (Steud.) Tzvelev) }\end{array}$ & $\begin{array}{l}\text { HP2B, } \\
\text { P2A }\end{array}$ \\
\hline Hordeum secalinum & Poaceae & Hordeum secalinum Schreb. & $\mathrm{P} 2 \mathrm{~A}$ \\
\hline Hydrocotyle asiatica & Araliaceae & Centella asiatica (L.) Urb. & $\mathrm{P} 5 \mathrm{~A}$ \\
\hline Hyppuris vulgaris & Hippuridaceae & Hippuris vulgaris $\mathrm{L}$. & P5A \\
\hline Imperata cylindrica & Poaceae & Imperata cylindrica (L.) Räusch. & $\mathrm{P} 2 \mathrm{~A}$ \\
\hline Ixora & Rubiaceae & Ixora sp. & P5A \\
\hline Lepturus cylindricus & Poaceae & Parapholis cylindrica (Willd.) Röser \& Tkach & $\mathrm{P} 2 \mathrm{~A}$ \\
\hline Lolium perenne & Poaceae & Lolium perenne L. & $\mathrm{P} 2 \mathrm{~A}$ \\
\hline Lycopodium clavatum & Lycopodiaceae & Lycopodium clavatum L. & P5A \\
\hline Malachra radiata & Malvaceae & Malachra radiata (L.) L. & $\mathrm{P} 5 \mathrm{~A}$ \\
\hline Marchantia polymorpha & Marchantiaceae & Marchantia polymorpha L. & P5A \\
\hline Montia minor & Montiaceae & $\begin{array}{l}\text { Montia fontana L. subsp. chondrosperma (Fenzl) } \\
\text { Walters }\end{array}$ & P5A \\
\hline Musa paradisiaca & Musaceae & Musa paradisiaca L. (pro sp.) & HP2 \\
\hline Ombrellifere & Apiaceae & Apiaceae Lindl. & P5A \\
\hline Panicum capillare & Poaceae & Panicum capillare $\mathrm{L}$. & $\mathrm{P} 2 \mathrm{~A}$ \\
\hline Panicum colonum & Poaceae & Echinochloa colona (L.) Link & $\mathrm{P} 2 \mathrm{~A}$ \\
\hline Panicum crus galli & Poaceae & Echinochloa crus-galli (L.) P.Beauv. subsp. crus-galli & $\mathrm{P} 2 \mathrm{~A}$ \\
\hline Papyrus & Cyperaceae & Cyperus papyrus L. & HP2B \\
\hline Parkinsonia aculeata & Fabaceae & Parkinsonia aculeata $\mathrm{L}$. & $\mathrm{P} 5 \mathrm{~A}$ \\
\hline Pavetta & Rubiaceae & Pavetta sp. & P5A \\
\hline Pennisetum cenchroides & Poaceae & Cenchrus ciliaris $\mathrm{L}$. & $\mathrm{P} 2 \mathrm{~A}$ \\
\hline Phalaris arundinacea & Poaceae & Phalaris arundinacea $\mathrm{L}$. & $\mathrm{P} 2 \mathrm{~A}$ \\
\hline Phalaris minor & Poaceae & Phalaris minor Retz. & $\mathrm{P} 2 \mathrm{~A}$ \\
\hline Phleum alpinum & Poaceae & Phleum alpinum $\mathrm{L}$. & P5A \\
\hline Phleum pratense & Poaceae & Phleum pratense $\mathrm{L}$. & $\mathrm{P} 2 \mathrm{~A}$ \\
\hline Phragmites communis & Poaceae & Phragmites australis (Cav.) Trin. ex Steud. & $\mathrm{P} 2 \mathrm{~A}$ \\
\hline Pisum maritimum & Fabaceae & $\begin{array}{l}\text { Lathyrus japonicus Willd. subsp. maritimus (L.) } \\
\text { P.W.Ball }\end{array}$ & P5A \\
\hline Plantago & Plantaginaceae & Plantago sp. & HP2B \\
\hline Poa annua & Poaceae & Poа аппиа $\mathrm{L}$. & $\begin{array}{l}\mathrm{HP} 2 \mathrm{~B} \\
\mathrm{P} 2 \mathrm{~A}\end{array}$ \\
\hline Poa compressa & Poaceae & Poa compressa $\mathrm{L}$. & $\mathrm{P} 2 \mathrm{~A}$ \\
\hline Poa nemoralis & Poaceae & Poa nemoralis L. & $\mathrm{P} 2 \mathrm{~A}$ \\
\hline Poa pratensis & Poaceae & Poa pratensis $\mathrm{L}$. & $\mathrm{P} 2 \mathrm{~A}$ \\
\hline
\end{tabular}




\begin{tabular}{|c|c|c|c|}
\hline Original indication & Family & Current name & Reference \\
\hline Poa trivialis & Poaceae & Poa trivialis $\mathrm{L}$. & $\mathrm{P} 2 \mathrm{~A}$ \\
\hline Polemonium caeruleum & Polemoniaceae & Polemonium caeruleum L. & P5A \\
\hline Polygon[um] aviculare & Polygonaceae & Polygonum aviculare L. & HP2B \\
\hline Polypogon maritimum & Poaceae & Polypogon maritimus Willd. & $\mathrm{P} 2 \mathrm{~A}$ \\
\hline Polypogon monspeliense & Poaceae & Polypogon monspeliensis (L.) Desf. & $\mathrm{P} 2 \mathrm{~A}$ \\
\hline Primula farinosa & Primulaceae & Primula farinosa $\mathrm{L}$. & P5A \\
\hline Primula magellanica & Primulaceae & Primula magellanica Lehm. & P5A \\
\hline Pteris longifolia & Pteridaceae & Pteris vittata $\mathrm{L}$. & HP2 \\
\hline Pterocarpus lunatus & Fabaceae & Machaerium lunatum (L.f.) Ducke & P5A \\
\hline Quercus & Fagaceae & Quercus sp. & HP2B \\
\hline Ranunculus aquatilis & Ranunculaceae & Ranunculus aquatilis L. & P5A \\
\hline Rhododendrum nivale & Ericaceae & Rhododendron nivale Hook.f. & P5A \\
\hline Rhyncosia caribaea & Fabaceae & Rhynchosia caribaea (Jacq.) DC. & P5A \\
\hline Rhyzophora mangle & Rhizophoraceae & Rhizophora mangle L. & P5A \\
\hline Robertia taraxacoides & Asteraceae & Robertia taraxacoides (Loisel.) DC. & HP2 \\
\hline Rubia & Rubiaceae & Rubia sp. & $\mathrm{P} 5 \mathrm{~A}$ \\
\hline Rumex aetnensis & Polygonaceae & $\begin{array}{l}\text { Rumex scutatus L. subsp. aetnensis (C.Presl) Cif. \& } \\
\text { Giacom. }\end{array}$ & HP2 \\
\hline Sagina procumbens & Caryophyllaceae & Sagina procumbens L. & P5A \\
\hline Samolus valerandi & Primulaceae & Samolus valerandi $\mathrm{L}$. & P5A \\
\hline Sauvagesia erecta & Ochnaceae & Sauvagesia erecta L. & P5A \\
\hline Saxifraga boussingaultii & Saxifragaceae & Saxifraga boussingaultii Brongn. & P5A \\
\hline Saxifraga indeterminée & Saxifragaceae & Saxifraga sp. & P5A \\
\hline Scirpus maritimus Linn. & Cyperaceae & Bolboschoenus maritimus (L.) Palla & $\mathrm{P} 2 \mathrm{~A}$ \\
\hline Scirpus Michelianus & Cyperaceae & Cyperus michelianus (L.) Delile & $\mathrm{P} 2 \mathrm{~A}$ \\
\hline Scirpus mucronatus & Cyperaceae & Schoenoplectiella mucronata (L.) J.Jung \& H.K.Choi & $\mathrm{P} 2 \mathrm{~A}$ \\
\hline Scirpus supinus & Cyperaceae & Schoenoplectiella supina (L.) Lye & $\mathrm{P} 2 \mathrm{~A}$ \\
\hline Senecio aetnensis & Asteraceae & $\begin{array}{l}\text { Senecio squalidus L. subsp. aethnensis (Jan ex DC.) } \\
\text { Greuter }\end{array}$ & HP2 \\
\hline Senecio vulgaris & Asteraceae & Senecio vulgaris $\mathrm{L}$. & P5A \\
\hline Serrafalcus mollis & Poaceae & Bromus hordeaceus L. & $\mathrm{P} 2 \mathrm{~A}$ \\
\hline Setaria glauca & Poaceae & Setaria pumila (Poir.) Roem. \& Schult. & $\mathrm{P} 2 \mathrm{~A}$ \\
\hline Setaria verticillata & Poaceae & Setaria verticillata (L.) P.Beauv. & $\mathrm{P} 2 \mathrm{~A}$ \\
\hline Setaria viridis & Poaceae & Setaria italica (L.) P.Beauv. subsp. viridis (L.) Thell. & $\mathrm{P} 2 \mathrm{~A}$ \\
\hline Sibbaldia procumbens & Rosaceae & Sibbaldia procumbens L. & P5A \\
\hline Silene acaulis & Caryophyllaceae & Silene acaulis $\mathrm{L}$. & HP2, P5A \\
\hline Sinapidendrum & Brassicaceae & Sinapidendron sp. & P5A \\
\hline Sisymbrium sophia & Brassicaceae & Descurainia sophia (L.) Webb ex Prantl & P5A \\
\hline Sporobolus pungens & Poaceae & Sporobolus virginicus (L.) Kunth & $\mathrm{P} 2 \mathrm{~A}$ \\
\hline Statice armeria & Plumbaginaceae & Armeria maritima (Mill.) Willd. & P5A \\
\hline
\end{tabular}




\begin{tabular}{|l|l|l|l|}
\hline Original indication & Family & Current name & Reference \\
\hline Stellaria media & Caryophyllaceae & Stellaria media L. & P5A \\
\hline Stellate & $\begin{array}{l}\text { Rubiaceae, pro } \\
\text { parte }\end{array}$ & Rubiaceae Juss., pro parte & P5A \\
\hline Stipa tortilis & Poaceae & Stipellula capensis (Thunb.) Röser \& H.R.Hamasha & P2A \\
\hline Taraxacum dens leonis & Asteraceae & Taraxacum campylodes G.E.Haglund & P5A \\
\hline Tetrapleura insularis & Apiaceae & Tornabenea insularis (Parl.) Parl. & P5A \\
\hline Torilis africana & Apiaceae & $\begin{array}{l}\text { Dasispermum capense } \text { (Lam.) Magee \& B.-E. van } \\
\text { Wyk. }\end{array}$ & \\
\hline Tragus racemosus & Poaceae & Tragus racemosus (L.) All. & P2A \\
\hline Trisetum andinum & Poaceae & $\begin{array}{l}\text { Koeleria spicata } \text { (L.) Barberá, Quintanar, Soreng \& } \\
\text { P.M. Peterson }\end{array}$ & P5A \\
\hline Trisetum flavescens & Poaceae & Trisetaria flavescens (L.) Baumg. & P2A \\
\hline Trisetum molle & Poaceae & $\begin{array}{l}\text { Koeleria spicata } \text { (L.) Barberá, Quintanar, Soreng \& } \\
\text { P.M. Peterson }\end{array}$ & P5A \\
\hline Trisetum subspicatum & Poaceae & $\begin{array}{l}\text { Koeleria spicata } \text { (L.) Barberá, Quintanar, Soreng \& } \\
\text { P.M. Peterson }\end{array}$ & P2A, P5A \\
\hline Urena americana & Urticaceae & Urena lobata L. & P5A \\
\hline Vulpia myuros & Poaceae & Festuca myuros L. & P2A \\
\hline Walteria americana & Malvaceae & Waltheria indica L. & P5A \\
\hline wilde Kartoffeln & Solanaceae & Solanum sp. sect. Petota Dumort. & HP2B \\
\hline Ximenia americana & Olacaceae & Ximenia americana L. & P5A \\
\hline
\end{tabular}

